\title{
Review of insecticide resistance and behavioral avoidance of vectors of human diseases in Thailand
}

Theeraphap Chareonviriyaphap ${ }^{1 *}$, Michael J Bangs ${ }^{2}$, Wannapa Suwonkerd ${ }^{3}$, Monthathip Kongmee ${ }^{4}$, Vincent Corbel ${ }^{1,5}$ and Ratchadawan Ngoen-Klan ${ }^{1}$

\begin{abstract}
Physiological resistance and behavioral responses of mosquito vectors to insecticides are critical aspects of the chemical-based disease control equation. The complex interaction between lethal, sub-lethal and excitation/ repellent ('excito-repellent') properties of chemicals is typically overlooked in vector management and control programs. The development of "physiological" resistance, metabolic and/or target site modifications, to insecticides has been well documented in many insect groups and disease vectors around the world. In Thailand, resistance in many mosquito populations has developed to all three classes of insecticidal active ingredients currently used for vector control with a majority being synthetic-derived pyrethroids. Evidence of low-grade insecticide resistance requires immediate countermeasures to mitigate further intensification and spread of the genetic mechanisms responsible for resistance. This can take the form of rotation of a different class of chemical, addition of a synergist, mixtures of chemicals or concurrent mosaic application of different classes of chemicals. From the gathered evidence, the distribution and degree of physiological resistance has been restricted in specific areas of Thailand in spite of long-term use of chemicals to control insect pests and disease vectors throughout the country. Most surprisingly, there have been no reported cases of pyrethroid resistance in anopheline populations in the country from 2000 to 2011. The precise reasons for this are unclear but we assume that behavioral avoidance to insecticides may play a significant role in reducing the selection pressure and thus occurrence and spread of insecticide resistance. The review herein provides information regarding the status of physiological resistance and behavioral avoidance of the primary mosquito vectors of human diseases to insecticides in Thailand from 2000 to 2011.
\end{abstract}

Keywords: Anopheles, Culex, Aedes, Control, Insecticide, Susceptibility, Behavior, Thailand

\section{Introduction}

A number of insect species can transmit pathogens to humans resulting in significant morbidity and mortality as well as placing a profound burden on human productivity and development. Transmission of these vector-borne diseases is related to the complex interplay of three primary components; pathogenicity/virulence of the infectious agent, vector competence (infectivity) and host (human) susceptibility. This transmission cycle is directly and indirectly driven by a diverse number of inter-related environmental factors. Successful control of human diseases

\footnotetext{
* Correspondence: faasthc@ku.ac.th

${ }^{1}$ Department of Entomology, Faculty of Agriculture, Kasetsart University, Bangkok 10900, Thailand

Full list of author information is available at the end of the article
}

requires an understanding of the interaction among these three components and the various other biological, environmental, and socio-economic factors that influence transmission. Such a task often requires or benefits from the full participation of both governmental and private sectors, sufficient numbers of trained personnel, adequate and sustained financial support, and a well-designed, evidencebased vector control program. Despite decades of organized vector control efforts, malaria, dengue, lymphatic filariasis and Japanese encephalitis, remain real threats in various areas of Thailand. One of the most effective means of prevention of these diseases involves vector control to reduce the risk of transmission. In some instances, this requires the use of various chemical compounds as larvicides applied to aquatic habitats and adulticides as outdoor space

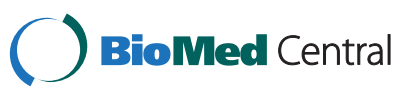


applications and indoor residual sprays (IRS), and the use of insecticide-treated bed nets [1-4] in order to reduce vector survival and density and thus human-vector contact.

At least four groups of synthetic compounds, organochlorine (DDT), organophosphates, carbamates and pyrethroids, have been extensively used in Thailand for the control of both agricultural pests and human/animal disease vectors. DDT was introduced for agricultural pest control in 1934 but was later banned from all agricultural use in 1983. For public health, the use of DDT was launched in 1949 in an indoor residual spray (IRS) pilot campaign to control malaria transmission after Thailand agreed to participate in the malaria eradication program outlined by the World Health Organization (WHO) [5-7]. However, the use of DDT began a gradual decline in use in the later decades and was completely removed for malaria control in the year 2000 due to its perceived adverse impact on the environment and declining public acceptance for indoor residual spraying [7-9]. Before 2000 , the extensive use of DDT resulted in the development of physiological resistance in populations of Anopheles aconitus, Anopheles culicifacies, Anopheles nivipes and Anopheles philippinensis, all non-malaria vector species in Thailand [8].

For many decades, various synthetic insecticidal compounds have been used extensively in the private sector, agri-business and in the national public health vector control programs in Thailand. The vast majority of compounds, by number and volume, are pyrethroid-based formulated combinations (Table 1). Synthetic pyrethroids have become the most popular and prevalent active ingredients for public health use due to their relatively low mammalian toxicity but high invertebrate potency at low levels, resulting in rapid immobilization ('knockdown') and killing [10]. Most of them have been used to control insect pests such as cockroaches, ants, bedbugs, and mosquitoes [11]. Compared to pyrethroids, relatively few organophosphate and carbamate-based insecticides remain available in the Thai public market. For example, the amount of pyrethroids used for dengue and malaria control in 2007 (excluding treated bed nets) was approximately 1,127 tons whereas that of OP and carbamates together was virtually nil [11].

The extensive use of pyrethroids for vector control has raised major concerns over the selection pressure induced by the insecticides on resistance gene mechanisms [4,12-15]. In addition to insecticides, topically applied repellents such as DEET ( $N, N$-diethyl-meta-toluamide), one of the most effective insect repellent active ingredients $[16,17]$, is available in most local markets and is used extensively to protect against biting mosquitoes and other insects (Table 1), despite potential negative health effects in humans associated with continuous or over application on the skin.
Insect resistance to insecticides has been observed for all classes of compounds, including microbial-based agents and insect growth regulators (hormone mimics) [18]. In general, response to insecticides can be categorized into two major types: physiological resistance and behavioral avoidance [19]. Physiological resistance is the ability of an insect population to survive exposure to a concentration of insecticide that would normally result in complete kill [1]. One or more mechanisms may be involved in physiological resistance, including alteration of target site nerve receptors (e.g., $k d r, R d l$ and $A c e .1 R$ ) and detoxification via increased enzyme activity of nonspecific esterases, glutathione $S$-transferases and P-450 mediated monooxygenases (mixed function oxidases) [20].

In contrast, behavioral avoidance (deterrence) is defined as the ability of an insect to move away (escape) from an insecticide-treated area, often without lethal consequence [4]. This type of response can be further divided into direct contact excitation (sometimes referred to as 'irritancy') and non-contact spatial repellency [12]. The term 'contact irritancy' involves an insect leaving an insecticide treated area only after making physical (tarsal) contact with the chemical, whereas 'spatial repellency' is when insects move away from the insecticide-treated area without making direct contact $[12,19]$. Lastly, some chemicals, such as DEET, can elicit a fourth action by effectively masking/jamming the presence of a host through the inhibition of odor-activated receptors [21].

The review herein has compiled information on the use of public health insecticides in Thailand since 2000 and summarizes the primary insecticidal and behavioral responses of disease vector mosquitoes of importance elicited by these chemicals [8]. This should assist in guiding national authorities in the rational and targetspecific use of insecticides for effective control of mosquito vectors.

\section{Review}

Insecticides used for the control of insect vectors and pests

Insecticides have been widely used to control both urban and peri-urban insect pests $[1,2,22]$. Although the use of DDT has been completely halted (banned) in many countries, recent allowances have been made for its renewed use in malaria control programs in a number of African nations because of its superior attributes compared to most alternative active ingredients [23]. The marked impact of DDT on mosquito populations in terms of both toxicity and modified behavioral responses that suppress disease transmission is well known despite lacking a clear understanding of the actual mechanisms and interactions at work in some instances. Most studies 
Table 1 Twenty-three commercial products and active ingredients for household pest control in Thailand (2012)

\begin{tabular}{|c|c|c|c|c|c|}
\hline Product name (Trade name) & Compounds & Concentration & (Continued) & & \\
\hline \multirow[t]{2}{*}{ Shieldtox (odorless I) } & Bioallethrin & $0.241 \% \mathrm{w} / \mathrm{w}$ & Kincho & Tetramethrin & $0.20 \% w / w$ \\
\hline & Bioresmethrin & $0.046 \% w / w$ & & Permethrin & $0.14 \% w / w$ \\
\hline \multirow[t]{2}{*}{ Shieldtox (odorless II) } & Prallethrin & $0.0729 \% \mathrm{w} / \mathrm{w}$ & JUMBO & Bioallethrin & $0.200 \% \mathrm{w} / \mathrm{w}$ \\
\hline & Phenothrin & $0.1003 \% \mathrm{w} / \mathrm{w}$ & & Deltamethrin & $0.012 \% \mathrm{w} / \mathrm{w}$ \\
\hline \multirow[t]{2}{*}{ Shieldtox (Ultra I) } & Bioallethrin & $0.209 \% \mathrm{w} / \mathrm{w}$ & & Permethrin & $0.100 \% \mathrm{w} / \mathrm{w}$ \\
\hline & Bioresmethrin & $0.039 \% \mathrm{w} / \mathrm{w}$ & Sheldrite & Permethrin & $0.25 \% w / w$ \\
\hline \multirow[t]{2}{*}{ Shieldtox (Ultra II) } & Tetramethrin & $0.230 \% \mathrm{w} / \mathrm{w}$ & & Bioallethrin & $0.10 \% w / w$ \\
\hline & Deltamethrin & $0.015 \% w / w$ & & Dichlovos & $0.5 \% \mathrm{w} / \mathrm{w}$ \\
\hline \multirow[t]{2}{*}{ Shieldtox (Ultra Green I) } & Tetramethrin & $0.230 \% w / w$ & Atsawin & Tetramethrin & $0.20 \% w / w$ \\
\hline & Deltamethrin & $0.015 \% \mathrm{w} / \mathrm{w}$ & & Permethrin & $0.10 \% w / w$ \\
\hline Shieldtox (Ultra Yellow 1) & Prallethrin & $0.055 \% \mathrm{w} / \mathrm{w}$ & GY-15 & DEET & $4 \%$ \\
\hline
\end{tabular}

Permethrin

Tetramethrin

$0.100 \% \mathrm{w} / \mathrm{w}$

Propoxur

$0.184 \% \mathrm{w} / \mathrm{w}$

Raid Insect Killer 4

Tetramethrin

$0.75 \% \mathrm{w} / \mathrm{w}$

Cypermethrin

$0.30 \% \mathrm{w} / \mathrm{w}$

Tetramethrin

$0.10 \% \mathrm{w} / \mathrm{w}$

Raid X-tra

Permethrin

$0.30 \% \mathrm{w} / \mathrm{w}$

$0.10 \% \mathrm{w} / \mathrm{w}$

Transfluthrin

$0.05 \% \mathrm{w} / \mathrm{w}$

Raid X-tra Plus

Prallethrin

$0.06 \% \mathrm{w} / \mathrm{w}$

Permethrin

$0.20 \% \mathrm{w} / \mathrm{w}$

Raid Insect Killer (water based)

Prallethrin

$0.06 \% \mathrm{w} / \mathrm{w}$

Permethrin

Raid (water based)

Tetramethrin

Allethrin

Permethrin

ARS

ARS 3

Tetramethrin

Dichlovos

Tetramethrin

Permethrin

Dichlovos

ARS (water based)

Allethrin

Tetramethrin

Permethrin

ARS Jet Pro

BYGON (Blue)

BYGON (Yellow)

BYGON (Green)
$0.24 \% \mathrm{w} / \mathrm{w}$

$0.35 \% \mathrm{w} / \mathrm{w}$

$0.10 \% \mathrm{w} / \mathrm{w}$

$0.10 \% \mathrm{w} / \mathrm{w}$

$0.07 \% \mathrm{w} / \mathrm{w}$

$0.50 \% \mathrm{w} / \mathrm{w}$

$0.02 \% \mathrm{w} / \mathrm{w}$

$0.10 \% \mathrm{w} / \mathrm{w}$

$0.05 \% \mathrm{w} / \mathrm{w}$

$0.06 \% \mathrm{w} / \mathrm{w}$

$0.06 \% \mathrm{w} / \mathrm{w}$

$0.18 \% \mathrm{w} / \mathrm{w}$

$0.20 \% \mathrm{w} / \mathrm{w}$

$0.10 \% \mathrm{w} / \mathrm{w}$

$0.025 \% \mathrm{w} / \mathrm{w}$

$0.040 \% \mathrm{w} / \mathrm{w}$

$0.04 \% \mathrm{w} / \mathrm{w}$

$0.025 \% \mathrm{w} / \mathrm{w}$

$0.025 \% \mathrm{w} / \mathrm{w}$

$0.500 \% \mathrm{w} / \mathrm{w}$

$0.500 \% \mathrm{w} / \mathrm{w}$
Table 1 Twenty-three commercial products and active ingredients for household pest control in Thailand (2012) (Continued)

on insecticides have focused exclusively on the direct toxicological effects of the molecule on mosquitoes whereas comparatively fewer investigations have accurately measured the behavioral responses resulting from sub-lethal exposure to the active ingredient $[12,19,24,25]$. Observations on behavioral responses of vectors began with the early use of DDT to control Anopheles mosquitoes [1], avoidance outcomes which resulted in the recognition of two different types of non-toxic actions: excitation and repellency, often termed together as 'excitorepellency' [1,4,26-33]. The importance (either benefit or drawbacks) of avoidance behavior without killing or reducing survival of the vector outright has produced plenty of debate and controversy for impact in controlling disease transmission $[4,32]$.

In Thailand, many compounds have been used for the control of medically important insects in both the private and public sectors. In the private sector, several dispensing designs for household pesticides are available [13,14], including space sprays (aerosols), released as a vapor phase (mosquito coils, electric mats), direct applications (creams), and residual liquids. Often, these various formats contain more than one active ingredient and include synergists to enhance knockdown response and effectiveness. Over $80 \%$ of the active ingredients currently used in homes are pyrethroids that are used in low concentrations in the form of aerosols. By market volume, both organophosphates and carbamates (Table 1) are used to a far lesser extent. The most common use for home-based insecticides is for control of mosquitoes and other flying insects (house and filth flies) followed by termites, ants, cockroaches, and bedbugs. Among the pyrethroids, permethrin, deltamethrin and cypermethrin are the predominant active ingredients used (Table 1). In the business sector, pest control operators (PCOs) with proper training and special licensure, allows professionals to apply a wider (and more toxic) variety of chemicals to control a broader range of pests (e.g.,
Imiprothrin

Cypermethrin

Cyfluthrin

Transfluthrin

Transfluthrin

Cyfluthrin

Cyfluthrin

Propoxur

Dichlorvos 
structural pests such as termites and ants). Such PCO training programs, under the support and guidance of the Food and Drug Administrative Office, Ministry of Public Health, has been carried out by the Department of Entomology, Faculty of Agriculture, Kasetsart University at least twice each year since 2003. In the public (government) sector, a wider array of chemicals are used for vector control purposes including organophosphates, carbamates, pyrethroids and so-called 'bio-rational' pesticides and biological agents, such as natural predators, bacterial toxins, insect growth regulators (hormone mimics) and botanical repellents, depending on the target species and circumstances $[2,8,34]$. For routine dengue vector control in Thailand, an organophosphate-based larvicide (temephos) has been commonly used for the control of Aedes aegypti larvae in container habitats since 1950 [8]. Although it remains mostly effective for Aedes larval control, evidence of temephos resistance in Ae. aegypti has recently been observed in some localities of Thailand [13]. The other organophosphates such as malathion, fenitrothion and pirimiphos-methyl were commonly used as either IRS or fogging agents before being replaced by pyrethroids [8]. In 1994, deltamethrin, a newer, more potent pyrethroid was introduced to Thailand for controlling indoor biting mosquitoes, including Ae. aegypti [8]. This compound remains the standard for the control of dengue vectors during dengue outbreaks although recent reports have identified deltamethrin resistance in several populations of Ae. aegypti [35]. Deltamethrin and permethrin have also been widely used in the malaria control program [36]. Deltamethrin is used for IRS once or twice a year, depending upon the intensity of malaria transmission in the area (based on endemic malaria zoning categories) determined by the Bureau of Vector Borne Disease [36]. Permethrin is still commonly used to impregnate fabric materials such as clothing, screens, blankets, and bed nets. These insecticide-treated materials can be more easily shipped to malaria endemic areas that may be difficult to access for spray teams because of relative isolation, poor roads and/ or on-going civil insurgency and security disruptions. For example, in the four southern-most provinces of Thailand, cases of malaria have risen to nearly 4,000 a year in the area adjacent to the Thai-Malaysian border where rebels have been engaging Thai authorities since 2004. Similarly, the number of malaria cases reported along the ThaiMyanmar border (e.g., Tak and Mae Hongsorn provinces) has been aggravated due to the presence of refugee camps and intense migration from the adjacent country where malaria is still highly prevalent [37].

\section{History and organization of the vector-borne disease control program}

Over the 60 year history of the malaria control in Thailand, a number of policy changes have occurred to adjust to new developments in technology and the shifting landscape of malaria epidemiology in the country. Since the implementation of the national malaria control program in the $1950 \mathrm{~s}$, malaria associated morbidity and mortality has been reduced dramatically in Thailand except along the border areas. However, in the last decade, a shortage of trained public health personnel and vector control specialists, together with flagging financial support has resulted in dramatic changes in the vector control policies in Thailand. In October 2002, the Department of Disease Control (DDC) reorganized the Vector-Borne Disease Control (VBDC) Program by merging the Malaria Control Unit (MCU) with other vectorborne disease control programs. The DDC was further consolidated to include other non-communicable diseases under its direction. The restructuring helped ease constraints on staffing, budget and equipment for all VBDC elements, and eliminated many of the redundancies and relatively high costs previously incurred by each of the former independent control programs. At the national level, the Bureau of Vector Borne Disease (BVBD) is under the direction of the DDC, in the Ministry of Public Health. The program comprises 12 regions under the direction of a Medical Officer, Director of the Office of Disease Prevention and Control (DPC). Under the DPC, the Vector-Borne Disease Section (VBDS) was set up to respond to the major vector-borne disease issues in each Regional DPC. In 2003, 39 Vector-Borne Disease Control (VBDC) Centers and 302 Vector-Borne Disease Control Units (VBDU) are set up at provincial and district levels, respectively, but recently these local VBD sectors were reduced to 38 VBDCs and 165 VBDUs, respectively. In addition, there are a number of district and sub-district municipalities that operate under the direction of the Ministry of Interior. In October 2011, the 12 DPC regional offices underwent further restructuring, and the VBDSs were merged with and renamed either Technical Support Sections or Emergency Response for Public Health Disasters, depending on the individual DPCs. This resulted in some confusion with the other Public Health Sectors with regard to knowing the correct individuals to contact for advice on insecticide usage and proper vector control application and monitoring techniques. This also included local district and sub-district administrative offices under the Ministry of Interior (Figure 1).

The Ministry of Interior is now responsible for developing their own policies for both vector and pest control activities, including planning and budget allocation. Little is done, however, in regard to pest control evaluation and monitoring. Moreover, each municipality has decision-making power for the local vector control operations, including budget allocations for purchasing insecticides. As a result, each local office can directly 


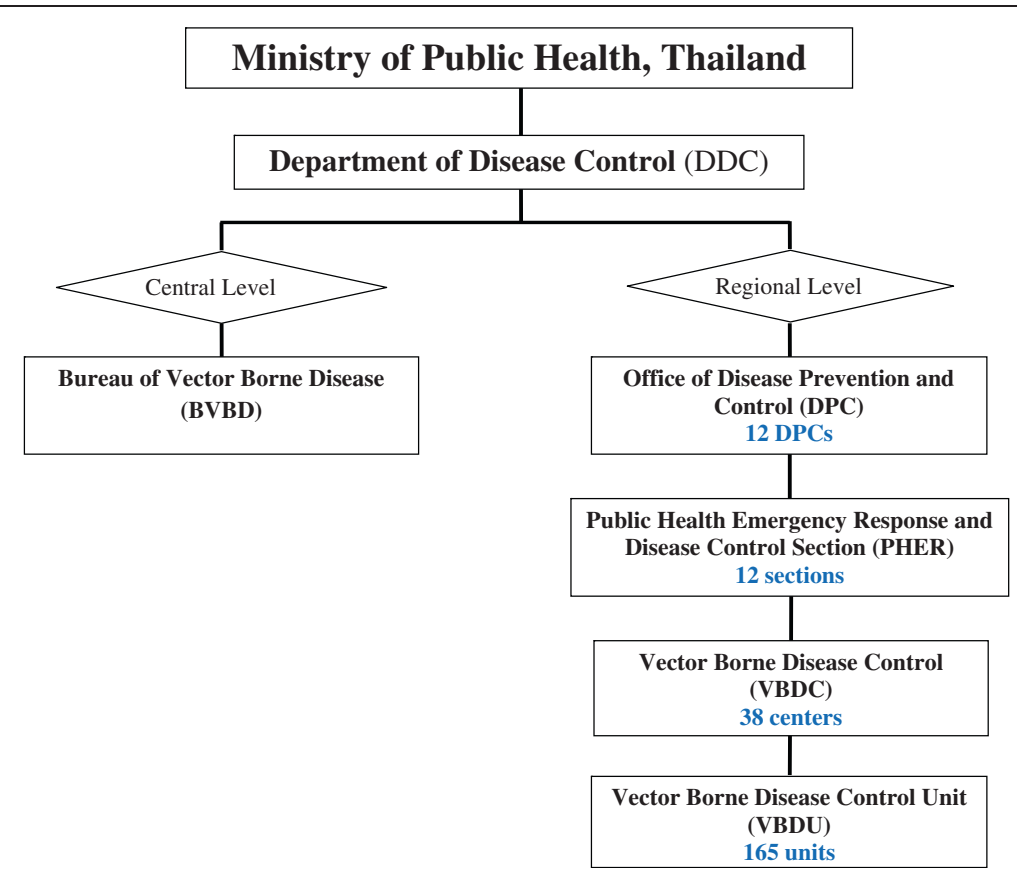

Figure 1 Diagram of the organization of the Vector-Borne Disease Control Program, Thailand.

approach an insecticide company without a clear policy and evidence-based rationale for insecticide choice from the Department of Disease Control, MOPH. The majority of products purchased are typically pyrethroids that include deltamethrin, permethrin, cypermethrin, and alphacypermethrin (Wannapa, personal communications).

\section{Mosquito-transmitted diseases in Thailand}

Thailand continues to face endemic transmission and the re-emergence of mosquito-borne diseases, principally malaria, dengue fever and dengue hemorrhagic fever (DF/DHF), lymphatic filariasis, Japanese encephalitis and more recently Chikungunya virus [36]. All these parasites and viruses are transmitted to humans by suitable vector mosquito species, some of which are capable of transmitting more than one disease pathogen [38]. The current distribution of these diseases in Thailand is presented in Figure 2. Like other countries in this region, malaria displays significant geographical heterogeneity and is exemplified by more intensified "border malaria", with most of the malaria cases concentrated along the Thai borders with Myanmar and Cambodia [7,39-41], and more recently with the upsurge of malaria near the border with northern Malaysia [36]. According to [42], $8 \%$ of the total Thai population ( $\sim 5$ million inhabitants) resides in high risk areas for malaria (i.e., 1 case per 1000 population), 42\% occupy lower risk areas (29 million population), while $50 \%$ are free from exposure to active malaria transmission (34 millions). The predominant malaria parasite species are Plasmodium falciparum and Plasmodium vivax but $P$. vivax has become slightly more prevalent than P. falciparum since 2000 [43].

Dengue viruses (Flavivirus) are responsible for one of the most prevalent vector-borne disease entities (DF/ DHF) of public health importance in much of the tropical and subtropical world [44]. Each year, between 50100 million dengue infections occur and nearly half the world population lives in the countries where dengue transmission is now endemic [45]. Much of urban Thailand is regarded as hyper-endemic for dengue infection with all four serotypes (DEN-1, DEN-2, DEN-3, DEN-4) regularly circulating and now occurring more commonly in rural areas. Unlike the majority of countries in the Southeast Asian Region, the total dengue cases reported in 2011 (78,337), $2010(68,386)$ and 2009 $(115,768)$ greatly exceeded that recorded in 2008 $(56,651)$ with an increase in the proportion of severe dengue cases being reported [46]. Chikungunya (an Alphavirus) is another arboviral disease, very similar in epidemiology and clinical presentation to dengue, but occupying different antigenic families, Togaviridae vs. Flaviviridae, respectively. Compared to dengue, chikungunya is a relatively uncommon reported disease and likely misdiagnosed as classic dengue infection where they co-exist $[47,48]$. Although periodic outbreaks occur throughout Africa and Southeast Asia, they are typically self-limiting infections and rarely result in severe disease. After years of apparent quiescence, chikungunya emerged in 2004 in Kenya and subsequently invaded the Indian Ocean islands of Comoros and Réunion in 2005 


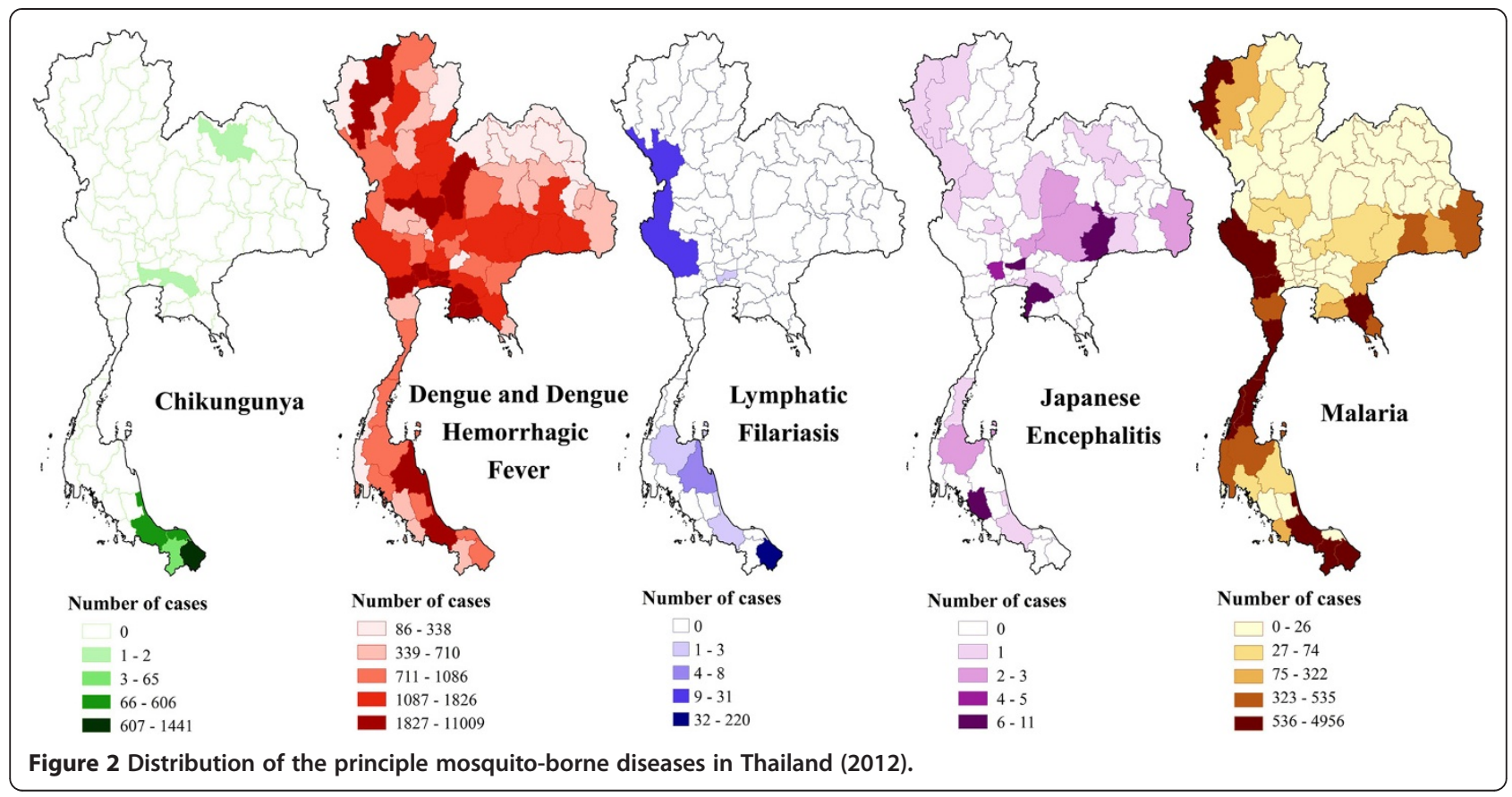

and rapidly spread to other islands of the Indian Ocean reaching India in 2006 where more than 1 million suspected cases were reported [48]. In the succeeding years, Sri Lanka, Indonesia, Singapore and Malaysia were affected, including reports of significant outbreaks of chikungunya in the south of Thailand in which 49,069 infections were reported with a notable increase of neurologic complications $[36,49]$.

Japanese encephalitis (JE), a Flavivirus, is the leading cause of viral encephalitis in Asian countries with approximately 30,000-50,000 cases reported annually, with case fatality rates between $0.3 \%$ and $60 \%$, depending on the age at time of infection and severity of symptoms [50]. In SE Asia, $\mathrm{JE}$ is rarely reported among travelers to countries where JE is endemic [51]. The number of overt cases has shown a sharp decline after mass immunization programs in the region. Transmission appears to be declining mostly in China, Japan, and Korea, but cases continue to be reported from Bangladesh, Myanmar, India, Nepal, Sri Lanka, Vietnam and Thailand. Japanese encephalitis was first recognized in Japan in the 1920s and is believed to have spread from India throughout Asia, Indonesia and more recently northern Australia and New Guinea Island [52,53]. Typically, JE occurs as outbreaks in the extensive rice growing areas of Asia associated with Culex tritaeniorhynchus, Culex gelidus, and Culex vishnui group mosquitoes [54,55], especially in Thailand [36]. Even through developing symptomatic illnesses, humans, cattle and horses are regarded as dead-end hosts and not involved in the natural transmission cycle that involves a bird-mosquito maintenance cycle and various species of aquatic wading birds (e.g., ardeid birds) and pigs as principal amplifying hosts.

Lymphatic filariasis (LF) is caused by several species of nematodes that reside in the lymphatic system of the vertebrate host and is estimated to collectively infect more than 120 million people worldwide [56,57]. This disease is known for its debilitating and disfiguring outcome in the more unfortunate, albeit relatively rare, cases. Filariasis remains a major public health threat in many Southeast Asian countries where it is endemic in 9 of 11 countries. In Thailand, both Wuchereria bancrofti and Brugia malayi are presently and widely distributed, particularly along the international borders. Wuchereria bancrofti has been found along the western ThaiMyanmar border, including the provinces of Ranong, Ratchaburi, Kanchanaburi, Tak and Mae Hongsorn [58]. The most common filarial parasite strain in Thailand is the nocturnal sub-periodic form with a distinct peak of

Table 2 List of Anopheles populations resistant to DDT in Thailand using the WHO standard contact assay (2000-2010)

\begin{tabular}{|c|c|c|c|c|}
\hline Species & Insecticide & Location (province-district) & Geographic coordinates (DMS) & Published sources \\
\hline Anopheles annularis & DDT & Chiang Mai-Chiang Dao & $19^{\circ} 32^{\prime} \mathrm{N} 98^{\circ} 54^{\prime} \mathrm{E}$ & Prapanthadara et al. 2000 [96] \\
\hline Anopheles annularis & DDT & Mae Hongsorn & $19^{\circ} 9^{\prime} \mathrm{N} 98^{\circ} 1^{\prime} \mathrm{E}$ & \\
\hline Anopheles minimus (A)* & DDT & Phrae & $18^{\circ} 6^{\prime} \mathrm{N} 100^{\circ} 16^{\prime} \mathrm{E}$ & \\
\hline
\end{tabular}

${ }^{*}$ An. minimus sensu strict. Note that resistance to DDT was also seen in non-vector anophelines of Thailand before 2000, Anopheles aconitus, Anopheles culicifacies, Anopheles nivipes and Anopheles philippinensis [8]. 
Table 3 Locations in Thailand with Aedes aegypti populations tested against insecticides using the WHO standard contact assay (2000-2011)

\begin{tabular}{|c|c|c|c|}
\hline Insecticides & Location (province-district) & Geographic coordinates (DMS) & Published sources \\
\hline \multirow[t]{16}{*}{ Cyfluthrin } & Nonthaburi-Mueang & $13^{\circ} 51^{\prime} 44^{\prime \prime} \mathrm{N} 100^{\circ} 30^{\prime} 48^{\prime \prime} \mathrm{E}$ & Paeporn et al. 2010 [99] \\
\hline & Saraburi-Mueang * & $14^{\circ} 31^{\prime} 38^{\prime \prime} \mathrm{N} 100^{\circ} 54^{\prime} 35^{\prime \prime} \mathrm{E}$ & \\
\hline & Singburi-Mueang & $14^{\circ} 53^{\prime} 18^{\prime \prime} \mathrm{N} 100^{\circ} 24^{\prime} 17^{\prime \prime} \mathrm{E}$ & \\
\hline & Phitsanulok-Mueang & $16^{\circ} 49^{\prime} 29^{\prime \prime} \mathrm{N} 100^{\circ} 15^{\prime} 34^{\prime \prime} \mathrm{E}$ & \\
\hline & Phichit-Mueang & $16^{\circ} 26^{\prime} 18^{\prime \prime} \mathrm{N} 100^{\circ} 21^{\prime} \mathrm{O}^{\prime \prime} \mathrm{E}$ & \\
\hline & Sukhothai-Mueang & $17^{\circ} 0^{\prime} 28^{\prime \prime} \mathrm{N} 99^{\circ} 49^{\prime} 23^{\prime \prime} \mathrm{E}$ & \\
\hline & Uttaradit-Mueang & $17^{\circ} 37^{\prime} 33^{\prime \prime} \mathrm{N} 100^{\circ} 5^{\prime} 48^{\prime \prime} \mathrm{E}$ & \\
\hline & Lamphun-Mueang & 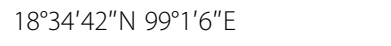 & \\
\hline & Chiang Mai-Mueang & 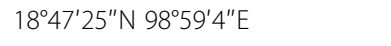 & \\
\hline & Chiang Rai-Mueang & $19^{\circ} 54^{\prime} 31^{\prime \prime N} 99^{\circ} 49^{\prime} 57^{\prime \prime} \mathrm{E}$ & \\
\hline & Khon Kean-Mueang & 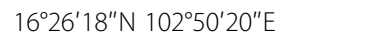 & \\
\hline & Prachinburi-Mueang & $14^{\circ} 3^{\prime} 2^{\prime \prime} \mathrm{N} 101^{\circ} 22^{\prime} \mathrm{O}^{\prime \prime} \mathrm{E}$ & \\
\hline & Sra Kaeo-Mueang & $13^{\circ} 48^{\prime} 52^{\prime \prime} \mathrm{N} 102^{\circ} 4^{\prime} 20^{\prime \prime} \mathrm{E}$ & Satimai 2010 [100] \\
\hline & Rayong-Mueang & $12^{\circ} 43^{\prime} 3^{\prime \prime} \mathrm{N} 101^{\circ} 23^{\prime} 31^{\prime \prime} \mathrm{E}$ & \\
\hline & Chanthaburi-Mueang & $12^{\circ} 36^{\prime} 40^{\prime \prime} \mathrm{N} 102^{\circ} 6^{\prime} 15^{\prime \prime} \mathrm{E}$ & \\
\hline & Trat-Mueang * & $12^{\circ} 13^{\prime} 54^{\prime \prime} \mathrm{N} 102^{\circ} 30^{\prime} 48^{\prime \prime} \mathrm{E}$ & \\
\hline \multirow[t]{2}{*}{ Cypermethrin } & Chiang Mai-Mae Tang & $19^{\circ} 10^{\prime} \mathrm{N} 98^{\circ} 54^{\prime} \mathrm{E}$ & Chareonviriyaphap et al. 2006 [101] \\
\hline & Kanchanaburi-Sai Yok & $14^{\circ} 17^{\prime} \mathrm{N} 99^{\circ} 11^{\prime} \mathrm{E}$ & \\
\hline \multirow[t]{25}{*}{ DDT } & Chiang Mai-Mae Tang & $19^{\circ} 8^{\prime} \mathrm{N} 98^{\circ} 51^{\prime} \mathrm{E}$ & Prapanthadara et al. 2002 [102] \\
\hline & Chiang Mai-Mae Tang & $19^{\circ} 11^{\prime} \mathrm{N} 98^{\circ} 54^{\prime} \mathrm{E}$ & Somboon et al. 2003 [93] \\
\hline & Chiang Mai-Mueang & $18^{\circ} 46^{\prime} \mathrm{N} 98^{\circ} 57^{\prime} \mathrm{E}$ & \\
\hline & Lampang-Mueang & $18^{\circ} 23^{\prime} \mathrm{N} 99^{\circ} 31^{\prime} \mathrm{E}$ & \\
\hline & Nan-Mueang & $18^{\circ} 47^{\prime} \mathrm{N} 100^{\circ} 43^{\prime} \mathrm{E}$ & \\
\hline & Chiang Mai-Mae Tang & $19^{\circ} 9^{\prime} \mathrm{N} 98^{\circ} 47^{\prime} \mathrm{E}$ & Lumjuan et al. 2005 [103] \\
\hline & Chiang Mai- Mae Tang & $19^{\circ} 8^{\prime} \mathrm{N} 98^{\circ} 51^{\prime} \mathrm{E}$ & Prapanthadara et al. 2005 [104] \\
\hline & Bangkok-Bang Khen & $13^{\circ} 52^{\prime} \mathrm{N} 100^{\circ} 35^{\prime} \mathrm{E}$ & Yaicharoen et al. 2005 [105] \\
\hline & Chiang Mai-Mae Tang & $19^{\circ} 9^{\prime} \mathrm{N} 98^{\circ} 52^{\prime} \mathrm{E}$ & Sathantriphop et al. 2006 [106] \\
\hline & Chonburi & $13^{\circ} 22^{\prime} 0^{\prime \prime} \mathrm{N} 100^{\circ} 58^{\prime} 60^{\prime \prime} \mathrm{E}$ & Rajatileka et al. 2008 [107] \\
\hline & Phang Nga & $8^{\circ} 28^{\prime} 0^{\prime \prime} \mathrm{N} 98^{\circ} 31^{\prime} 60^{\prime \prime} \mathrm{E}$ & \\
\hline & Phang Nga-Thap Pud & 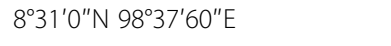 & \\
\hline & Chiang Mai-Mae Tang & $19^{\circ} 14^{\prime} \mathrm{N} 98^{\circ} 59^{\prime} \mathrm{E}$ & Thanispong et al. 2008 [14] \\
\hline & Pathum Thani-Lad Lumkeaw & $14^{\circ} 02^{\prime} \mathrm{N} 100^{\circ} 24^{\prime} \mathrm{E}$ & \\
\hline & Chiang Mai-Mueang & $18^{\circ} 47^{\prime} \mathrm{N} 99^{\circ} 00^{\prime} \mathrm{E}$ & \\
\hline & Kanchanaburi-Sai Yok & $14^{\circ} 20^{\prime} \mathrm{N} 98^{\circ} 59^{\prime} \mathrm{E}$ & \\
\hline & Nonthaburi-Mueang & $13^{\circ} 53^{\prime} \mathrm{N} 100^{\circ} 29^{\prime} \mathrm{E}$ & \\
\hline & Songkhla-Mueang & $7^{\circ} 11^{\prime} \mathrm{N} 100^{\circ} 35^{\prime} \mathrm{E}$ & \\
\hline & Satun-Mueang & $6^{\circ} 37^{\prime} \mathrm{N} 100^{\circ} 03^{\prime} \mathrm{E}$ & \\
\hline & Bangkok-Chatuchak & $13^{\circ} 50^{\prime} \mathrm{N} 100^{\circ} 34^{\prime} \mathrm{E}$ & \\
\hline & Lampang-Mueang & $18^{\circ} 17^{\prime} \mathrm{N} 99^{\circ} 29^{\prime} \mathrm{E}$ & \\
\hline & Tak-Mae Sot & $16^{\circ} 46^{\prime} \mathrm{N} 98^{\circ} 34^{\prime} \mathrm{E}$ & \\
\hline & Khon Kean-Mueang & $16^{\circ} 25^{\prime} \mathrm{N} 102^{\circ} 50^{\prime} \mathrm{E}$ & \\
\hline & Surat Thani-Mueang & $9^{\circ} 08^{\prime} \mathrm{N} 99^{\circ} 20^{\prime} \mathrm{E}$ & \\
\hline & Nakhon Sawan-Mueang & $15^{\circ} 42^{\prime} \mathrm{N} 100^{\circ} 08^{\prime} \mathrm{E}$ & \\
\hline
\end{tabular}


Table 3 Locations in Thailand with Aedes aegypti populations tested against insecticides using the WHO standard contact assay (2000-2011) (Continued)

\begin{tabular}{|c|c|c|c|}
\hline & Kamphaeng Phet & $16^{\circ} 28^{\prime} \mathrm{O}^{\prime \prime} \mathrm{N} 99^{\circ} 30^{\prime} \mathrm{O}^{\prime \prime} \mathrm{E}$ & \\
\hline & Phang Nga-Takua Pa & $8^{\circ} 52^{\prime} \mathrm{O}^{\prime \prime N} 98^{\circ} 20^{\prime} 60^{\prime \prime} \mathrm{E}$ & \\
\hline & Phuket & $7^{\circ} 52^{\prime} 60^{\prime \prime} \mathrm{N} 98^{\circ} 24^{\prime} 0^{\prime \prime} \mathrm{E}$ & \\
\hline \multirow[t]{41}{*}{ Deltamethrin } & Chiang Mai-Mueang & $18^{\circ} 46^{\prime} \mathrm{N} 98^{\circ} 57^{\prime} \mathrm{E}$ & Somboon et al. 2003 [93] \\
\hline & Nan-Muang & $18^{\circ} 47^{\prime} \mathrm{N} 100^{\circ} 43^{\prime} \mathrm{E}$ & \\
\hline & Ratchaburi-Pongsawai & $13^{\circ} 32^{\prime} 43^{\prime \prime} \mathrm{N} 99^{\circ} 51^{\prime} 7 " \mathrm{E}$ & Paeporn et al. 2004 [34] \\
\hline & Ratchaburi-KhuBua & $13^{\circ} 28^{\prime} 53^{\prime \prime} \mathrm{N} 99^{\circ} 49^{\prime} 21^{\prime \prime} \mathrm{E}$ & \\
\hline & Chiang Mai-Mae Tang & $19^{\circ} 10^{\prime} \mathrm{N} 98^{\circ} 54^{\prime} \mathrm{E}$ & Chareonviriyaphap et al. 2006 [101] \\
\hline & Bangkok-Bang Khen & $13^{\circ} 52^{\prime} 26^{\prime \prime} \mathrm{N} 100^{\circ} 35^{\prime} 47^{\prime \prime} \mathrm{E}$ & Yaicharoen et al. 2005 [105] \\
\hline & Bangkok-Hauykwang & $13^{\circ} 4^{\prime} 47.4^{\prime \prime} \mathrm{N} 100^{\circ} 3^{\prime} 52.3^{\prime \prime} \mathrm{E}$ & Jirakanjanakit et al. 2007 [13] \\
\hline & Bangkok-Laksi & $13^{\circ} 5^{\prime} 28.2^{\prime \prime} \mathrm{N} 100^{\circ} 3^{\prime} 41.2^{\prime \prime} \mathrm{E}$ & \\
\hline & Bangkok-Ladkrabang & $13^{\circ} 4^{\prime} 47.5^{\prime \prime} \mathrm{N} 100^{\circ} 4^{\prime} 23.6^{\prime \prime} \mathrm{E}$ & \\
\hline & Bangkok-Rasburana & $13^{\circ} 3^{\prime} 59.2^{\prime \prime} \mathrm{N} 100^{\circ} 3^{\prime} 58.8^{\prime \prime} \mathrm{E}$ & \\
\hline & Chonburi-Panusnikom & $13^{\circ} 2^{\prime} 2.9^{\prime \prime} \mathrm{N} 101^{\circ} 1^{\prime} 5.3^{\prime \prime} \mathrm{E}$ & \\
\hline & Chonburi-Banglamung & $12^{\circ} 5^{\prime} 42.2^{\prime \prime} \mathrm{N} 100^{\circ} 5^{\prime} 32.2^{\prime \prime} \mathrm{E}$ & \\
\hline & Nakhon Sawan-Taklee & $15^{\circ} 1^{\prime} 53^{\prime \prime} \mathrm{N} 100^{\circ} 1^{\prime} 48.2^{\prime \prime} \mathrm{E}$ & \\
\hline & Chanthaburi-Mueang & $13^{\circ} 19^{\prime} \mathrm{N} 100^{\circ} 55^{\prime} \mathrm{E}$ & \\
\hline & Nakhon Ratchasima-Kornburi & $14^{\circ} 3^{\prime} 50^{\prime \prime} \mathrm{N} 102^{\circ} 1^{\prime} 45.5^{\prime \prime} \mathrm{E}$ & \\
\hline & Nakhon Ratchasima-Senagsang & $14^{\circ} 2^{\prime} 35.2^{\prime \prime} \mathrm{N} 102^{\circ} 2^{\prime} 45.6^{\prime \prime}$ & \\
\hline & Nakhon Sawan-Mae Pern & $15^{\circ} 3^{\prime} 21^{\prime \prime} \mathrm{N} 99^{\circ} 2^{\prime} 48^{\prime \prime} \mathrm{E}$ & \\
\hline & Nakhon Sawan-Mae Wong & 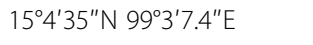 & \\
\hline & Nakhon Ratchasrima-Kornburi & $14^{\circ} 31^{\prime} 24^{\prime \prime} \mathrm{N} 102^{\circ} 14^{\prime} 54^{\prime \prime} \mathrm{E}$ & \\
\hline & Bangkok-Hauykwang & $13^{\circ} 45^{\prime} 54^{\prime \prime} \mathrm{N} 100^{\circ} 34^{\prime} 39^{\prime \prime} \mathrm{E}$ & Pethuan et al. 2007 [108] \\
\hline & Bangkok-Laksi & $13^{\circ} 53^{\prime} 15^{\prime \prime} \mathrm{N} 100^{\circ} 34^{\prime} 44^{\prime \prime} \mathrm{E}$ & \\
\hline & Chonburi-Panusnikom & $13^{\circ} 27^{\prime} 6^{\prime \prime} \mathrm{N} 101^{\circ} 10^{\prime} 36^{\prime \prime} \mathrm{E}$ & \\
\hline & Chonburi-Banglamung & $12^{\circ} 58^{\prime} 36^{\prime \prime} \mathrm{N} 100^{\circ} 54^{\prime} 48^{\prime \prime} \mathrm{E}$ & \\
\hline & Nakhon Sawan-Taklee & $15^{\circ} 15^{\prime} 47^{\prime \prime} \mathrm{N} 100^{\circ} 20^{\prime} 37^{\prime \prime} \mathrm{E}$ & \\
\hline & Nakhon Ratchasrima-Serngsang & $14^{\circ} 25^{\prime} 34^{\prime \prime} \mathrm{N} 102^{\circ} 27^{\prime} 38^{\prime \prime} \mathrm{E}$ & \\
\hline & Nakhon Sawan-Mae Pern & $15^{\circ} 39^{\prime} 28^{\prime \prime} \mathrm{N} 99^{\circ} 28^{\prime} 9^{\prime \prime} \mathrm{E}$ & \\
\hline & Nakhon Sawan-Mae Wong & $15^{\circ} 46^{\prime} 52^{\prime \prime} \mathrm{N} 99^{\circ} 31^{\prime} 9^{\prime \prime} \mathrm{E}$ & \\
\hline & Chanthaburi-Makham * & $12^{\circ} 40^{\prime} 25^{\prime \prime} \mathrm{N} 102^{\circ} 11^{\prime} 48^{\prime \prime} \mathrm{E}$ & Narksuwan et al. 2008 [109] \\
\hline & Phetchaburi-Tha Yang * & $12^{\circ} 58^{\prime} 24^{\prime \prime} \mathrm{N} 99^{\circ} 53^{\prime} 16^{\prime \prime} \mathrm{E}$ & \\
\hline & Buri Ram-Cham ni * & $14^{\circ} 47^{\prime} 18^{\prime \prime} \mathrm{N} 102^{\circ} 50^{\prime} 30^{\prime \prime} \mathrm{E}$ & \\
\hline & Loei-Nong Hin * & $17^{\circ} 7^{\prime} 24^{\prime \prime} \mathrm{N} 101^{\circ} 51^{\prime} 30^{\prime \prime} \mathrm{E}$ & \\
\hline & Trang-Sikao * & $07^{\circ} 34^{\prime} 18^{\prime \prime} \mathrm{N} 99^{\circ} 20^{\prime} 42^{\prime \prime} \mathrm{E}$ & \\
\hline & Chum phon-Lang Suan & $09^{\circ} 56^{\prime} 42^{\prime \prime} \mathrm{N} 99^{\circ} 4^{\prime} 42^{\prime \prime} \mathrm{E}$ & \\
\hline & Phetchabun-Wichian Buri * & $15^{\circ} 39^{\prime} 26^{\prime \prime} \mathrm{N} 101^{\circ} 6^{\prime} 24^{\prime \prime} \mathrm{E}$ & \\
\hline & Kalasin-Yangtalat * & $16^{\circ} 24^{\prime} 8^{\prime \prime} \mathrm{N} 103^{\circ} 22^{\prime} 23^{\prime \prime} \mathrm{E}$ & \\
\hline & Lampang-Thoen * & $17^{\circ} 36^{\prime} 42^{\prime \prime} \mathrm{N} 99^{\circ} 12^{\prime} 57^{\prime \prime} \mathrm{E}$ & \\
\hline & Uthai Thani-Thap Than* & $15^{\circ} 29^{\prime} 12^{\prime \prime} \mathrm{N} 99^{\circ} 49^{\prime} 54^{\prime \prime} \mathrm{E}$ & \\
\hline & Suphanburi-Bang plama * & $14^{\circ} 24^{\prime} 8^{\prime \prime} \mathrm{N} 100^{\circ} 9^{\prime} 16^{\prime \prime} \mathrm{E}$ & \\
\hline & Ratchaburi-Damneon Saduak & $13^{\circ} 31^{\prime} 6^{\prime \prime} \mathrm{N} 99^{\circ} 57^{\prime} 18^{\prime \prime} \mathrm{E}$ & \\
\hline & Ayutthaya-Phak Hai * & $14^{\circ} 27^{\prime} 30^{\prime \prime} \mathrm{N} 100^{\circ} 22^{\prime} 12^{\prime \prime} \mathrm{E}$ & \\
\hline & Nonthaburi-Mueang & 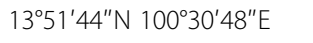 & Paeporn et al. 2010 [99] \\
\hline
\end{tabular}


Table 3 Locations in Thailand with Aedes aegypti populations tested against insecticides using the WHO standard contact assay (2000-2011) (Continued)

\begin{tabular}{|c|c|c|c|}
\hline & Nakhon Pathom-Mueang & $13^{\circ} 49^{\prime} 11^{\prime \prime} \mathrm{N} 100^{\circ} 3^{\prime} 57^{\prime \prime} \mathrm{E}$ & \\
\hline & Sra Kaeo-Mueang * & $13^{\circ} 48^{\prime} 53^{\prime \prime} \mathrm{N} 102^{\circ} 4^{\prime} 19^{\prime \prime} \mathrm{E}$ & \\
\hline & Saraburi-Mueang * & $14^{\circ} 31^{\prime} 38^{\prime \prime} \mathrm{N} 100^{\circ} 54^{\prime} 35^{\prime \prime} \mathrm{E}$ & \\
\hline & Lopburi-Mueang & 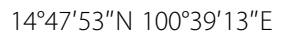 & \\
\hline & Suphanburi-Mueang & $14^{\circ} 29^{\prime} 4^{\prime \prime} \mathrm{N} 100^{\circ} 7^{\prime} 25^{\prime \prime} \mathrm{E}$ & \\
\hline & Angthong-Mueang & $14^{\circ} 35^{\prime} 19^{\prime \prime} \mathrm{N} 100^{\circ} 27^{\prime} 12^{\prime \prime} \mathrm{E}$ & \\
\hline & Singburi-Mueang & $14^{\circ} 53^{\prime} 18^{\prime \prime} \mathrm{N} 100^{\circ} 24^{\prime} 17^{\prime \prime} \mathrm{E}$ & \\
\hline & Kanchanaburi-Mueang & $14^{\circ} 0^{\prime} 12^{\prime \prime} \mathrm{N} 99^{\circ} 33^{\prime} \mathrm{O}^{\prime \prime} \mathrm{E}$ & \\
\hline & Phitsanulok-Mueang & $16^{\circ} 49^{\prime} 29^{\prime \prime} \mathrm{N} 100^{\circ} 15^{\prime} 34^{\prime \prime} \mathrm{E}$ & \\
\hline & Phichit-Mueang & $16^{\circ} 26^{\prime} 18^{\prime \prime} \mathrm{N} 100^{\circ} 21^{\prime} \mathrm{O}^{\prime \prime} \mathrm{E}$ & \\
\hline & Sukhothai-Mueang & $17^{\circ} 0^{\prime} 28^{\prime \prime} \mathrm{N} 99^{\circ} 49^{\prime} 23^{\prime \prime} \mathrm{E}$ & \\
\hline & Uttaradit-Mueang & $17^{\circ} 37^{\prime} 33^{\prime \prime} \mathrm{N} 100^{\circ} 5^{\prime} 48^{\prime \prime} \mathrm{E}$ & \\
\hline & Lamphun-Mueang & $18^{\circ} 34^{\prime} 42^{\prime \prime} \mathrm{N} 99^{\circ} 1^{\prime} 6^{\prime \prime} \mathrm{E}$ & \\
\hline & Uthaithani-Mueang * & $15^{\circ} 22^{\prime} 46^{\prime \prime} \mathrm{N} 100^{\circ} 1^{\prime} 29^{\prime \prime} \mathrm{E}$ & \\
\hline & Chiang Mai-Mueang & $18^{\circ} 47^{\prime} 25^{\prime \prime} \mathrm{N} 98^{\circ} 59^{\prime} 4^{\prime \prime} \mathrm{E}$ & \\
\hline & Chiang Rai-Mueang * & $19^{\circ} 54^{\prime} 31^{\prime \prime N} 99^{\circ} 49^{\prime} 57^{\prime \prime} \mathrm{E}$ & \\
\hline & Khon Kean-Mueang & 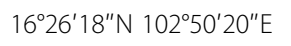 & \\
\hline & Udonthani-Mueang & $17^{\circ} 24^{\prime} 54^{\prime \prime} \mathrm{N} 102^{\circ} 47^{\prime} 12^{\prime \prime} \mathrm{E}$ & \\
\hline & Chai yaphum-Mueang & $15^{\circ} 48^{\prime} 35^{\prime \prime} \mathrm{N} 102^{\circ} 1^{\prime} 13^{\prime \prime} \mathrm{E}$ & \\
\hline & Nakhon Nayok-Mueang* & $14^{\circ} 12^{\prime} 13^{\prime \prime} \mathrm{N} 101^{\circ} 13^{\prime} 2^{\prime \prime} \mathrm{E}$ & \\
\hline & Rayong-Mueang * & $12^{\circ} 40^{\prime} 6^{\prime \prime} \mathrm{N} 101^{\circ} 16^{\prime} 30^{\prime \prime} \mathrm{E}$ & \\
\hline & Prachinburi-Mueang & $14^{\circ} 3^{\prime} 2^{\prime \prime} \mathrm{N} 101^{\circ} 22^{\prime} \mathrm{O}^{\prime \prime} \mathrm{E}$ & \\
\hline & Chonburi-Mueang & $13^{\circ} 21^{\prime} 43^{\prime \prime} \mathrm{N} 100^{\circ} 58^{\prime} 45^{\prime \prime} \mathrm{E}$ & \\
\hline & Sra Kaeo-Mueang * & $13^{\circ} 48^{\prime} 52^{\prime \prime} \mathrm{N} 102^{\circ} 4^{\prime} 20^{\prime \prime} \mathrm{E}$ & Satimai 2010 [100] \\
\hline & Rayong-Mueang & 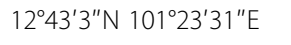 & \\
\hline & Chanthaburi-Mueang & $12^{\circ} 36^{\prime} 40^{\prime \prime} \mathrm{N} 102^{\circ} 6^{\prime} 15^{\prime \prime} \mathrm{E}$ & \\
\hline & Trat-Mueang * & $12^{\circ} 13^{\prime} 54^{\prime \prime} \mathrm{N} 102^{\circ} 30^{\prime} 48^{\prime \prime} \mathrm{E}$ & \\
\hline & Bangkok-Kannayaw & $13^{\circ} 50^{\prime} \mathrm{N} 100^{\circ} 40^{\prime} \mathrm{E}$ & Chuaycharoensuk et al. 2011 [35] \\
\hline & Chanthaburi-Mueang & $12^{\circ} 39^{\prime} \mathrm{N} 102^{\circ} 7^{\prime} \mathrm{E}$ & \\
\hline & Chonburi-Mueang & $13^{\circ} 19^{\prime} \mathrm{N} 100^{\circ} 55^{\prime} \mathrm{E}$ & \\
\hline & Khon Kean-Mueang & $16^{\circ} 19^{\prime} \mathrm{N} 102^{\circ} 47^{\prime} \mathrm{E}$ & \\
\hline & Udonthani-Wungsammor & $15^{\circ} 54^{\prime} \mathrm{N} 103^{\circ} 28^{\prime} \mathrm{E}$ & \\
\hline & Nakhon Sawan-Mueang & $15^{\circ} 40^{\prime} \mathrm{N} 100^{\circ} 05^{\prime} \mathrm{E}$ & \\
\hline & Tak-Mae Sot & $16^{\circ} 43^{\prime} \mathrm{N} 98^{\circ} 34^{\prime} \mathrm{E}$ & \\
\hline & Chumphon-Mueang & $10^{\circ} 30^{\prime} \mathrm{N} 99^{\circ} 07^{\prime} \mathrm{E}$ & \\
\hline & Prachuap Khiri Khan-Hua Hin & $12^{\circ} 33^{\prime} \mathrm{N} 99^{\circ} 53^{\prime} \mathrm{E}$ & \\
\hline & Songkhla-Namom & $06^{\circ} 54^{\prime} \mathrm{N} 100^{\circ} 32^{\prime} \mathrm{E}$ & \\
\hline & Songkhla-Sadao & $06^{\circ} 45^{\prime} \mathrm{N} 100^{\circ} 24^{\prime} \mathrm{E}$ & \\
\hline & Songkhla-Had Yai & $07^{\circ} 00^{\prime} \mathrm{N} 100^{\circ} 27^{\prime} \mathrm{E}$ & \\
\hline & Surat Thani-Mueang & $09^{\circ} 02^{\prime} \mathrm{N} 99^{\circ} 22^{\prime} \mathrm{E}$ & \\
\hline Etofenprox & Nan-Tha Wang Pha & $19^{\circ} 7^{\prime} \mathrm{N} 100^{\circ} 43^{\prime} \mathrm{E}$ & Somboon et al. 2003 [93] \\
\hline \multirow[t]{3}{*}{ Fenitrothion } & Nakhon Sawan-Mueang & $15^{\circ} 4^{\prime} 47^{\prime \prime} \mathrm{N} 100^{\circ} 34.7^{\prime \prime} \mathrm{E}$ & \multirow[t]{3}{*}{ Jirakanjanakit et al. 2007 [13] } \\
\hline & Nakhon Sawan-Krok Pra & $15^{\circ} 3^{\prime} 12^{\prime \prime} \mathrm{N} 100^{\circ} 33.7^{\prime \prime} \mathrm{E}$ & \\
\hline & Nakhon Ratchasima-Prathai & $15^{\circ} 3^{\prime} 56^{\prime \prime} \mathrm{N} 102^{\circ} 3^{\prime} 45.7^{\prime \prime} \mathrm{E}$ & \\
\hline
\end{tabular}


Table 3 Locations in Thailand with Aedes aegypti populations tested against insecticides using the WHO standard contact assay (2000-2011) (Continued)

\begin{tabular}{|c|c|c|c|}
\hline & Nakhon Ratchasima-Kagsanamnang & $15^{\circ} 4^{\prime} 14^{\prime \prime} \mathrm{N} 102^{\circ} 1^{\prime} 36.9^{\prime \prime} \mathrm{E}$ & \\
\hline & Nakhon Ratchasima-Seekhew & $14^{\circ} 5^{\prime} 19.5^{\prime \prime} \mathrm{N} 101^{\circ} 4^{\prime} 28.8^{\prime \prime} \mathrm{E}$ & \\
\hline & Nakhon Ratchasima-Senagsang & $14^{\circ} 2^{\prime} 35.2^{\prime \prime} \mathrm{N} 102^{\circ} 2^{\prime} 45.6^{\prime \prime} \mathrm{E}$ & \\
\hline & Nakhon Sawan-Mae Pern & $15^{\circ} 3^{\prime} 21^{\prime \prime} \mathrm{N} 99^{\circ} 2^{\prime} 48^{\prime \prime} \mathrm{E}$ & \\
\hline & Nakhon Sawan-Mae Wong & $15^{\circ} 4^{\prime} 35^{\prime \prime} \mathrm{N} 99^{\circ} 3^{\prime} 7.4^{\prime \prime} \mathrm{E}$ & \\
\hline & Nakhon Ratchasrima-Seekhew & $14^{\circ} 53^{\prime} 30^{\prime \prime} \mathrm{N} 101^{\circ} 43^{\prime} 24^{\prime \prime} \mathrm{E}$ & Pethuan et al. 2007 [108] \\
\hline & Nakhon Ratchasrima-Prathai & $15^{\circ} 32^{\prime} \mathrm{O}^{\prime \prime} \mathrm{N} 102^{\circ} 43^{\prime} 22^{\prime \prime} \mathrm{E}$ & \\
\hline & Nakhon Ratchasrima-Kangsanamnang & $15^{\circ} 45^{\prime} 0^{\prime \prime} \mathrm{N} 102^{\circ} 15^{\prime} 17^{\prime \prime} \mathrm{E}$ & \\
\hline & Nakhon Sawan-Mueang & $15^{\circ} 44^{\prime} 15^{\prime \prime} \mathrm{N} 100^{\circ} 5^{\prime} 21^{\prime \prime} \mathrm{E}$ & \\
\hline & Nakhon Sawan-Krok Pra & $15^{\circ} 34^{\prime} 48^{\prime \prime} \mathrm{N} 100^{\circ} 0^{\prime} 13^{\prime \prime} \mathrm{E}$ & \\
\hline & Nakhon Ratchasrima-Serngsang & $14^{\circ} 25^{\prime} 34^{\prime \prime} \mathrm{N} 102^{\circ} 27^{\prime} 38^{\prime \prime} \mathrm{E}$ & \\
\hline & Nakhon Sawan-Mae Pern & $15^{\circ} 39^{\prime} 28^{\prime \prime} \mathrm{N} 99^{\circ} 28^{\prime} 9^{\prime \prime} \mathrm{E}$ & \\
\hline & Nakhon Sawan-Mae Wong & $15^{\circ} 46^{\prime} 52^{\prime \prime} \mathrm{N} 99^{\circ} 31^{\prime} 9^{\prime \prime} \mathrm{E}$ & \\
\hline & Nonthaburi -Mueang & $13^{\circ} 51^{\prime} 44^{\prime \prime} \mathrm{N} 100^{\circ} 30^{\prime} 48^{\prime \prime} \mathrm{E}$ & Paeporn et al. 2010 [99] \\
\hline & Sra Kaeo-Mueang * & $13^{\circ} 48^{\prime} 53^{\prime \prime} \mathrm{N} 102^{\circ} 4^{\prime} 19^{\prime \prime} \mathrm{E}$ & \\
\hline & Saraburi-Mueang * & $14^{\circ} 31^{\prime} 38^{\prime \prime} \mathrm{N} 100^{\circ} 54^{\prime} 35^{\prime \prime} \mathrm{E}$ & \\
\hline & Lopburi-Mueang & $14^{\circ} 47^{\prime} 53^{\prime \prime} \mathrm{N} 100^{\circ} 39^{\prime} 13^{\prime \prime}$ & \\
\hline & Suphanburi-Mueang & $14^{\circ} 29^{\prime} 4^{\prime \prime} \mathrm{N} 100^{\circ} 7^{\prime} 25^{\prime \prime} \mathrm{E}$ & \\
\hline & Angthong-Mueang & $14^{\circ} 35^{\prime} 19^{\prime \prime} \mathrm{N} 100^{\circ} 27^{\prime} 12^{\prime \prime} \mathrm{E}$ & \\
\hline & Singburi-Mueang * & $14^{\circ} 53^{\prime} 18^{\prime \prime} \mathrm{N} 100^{\circ} 24^{\prime} 17^{\prime \prime} \mathrm{E}$ & \\
\hline & Phitsanulok-Mueang * & $16^{\circ} 49^{\prime} 29^{\prime \prime} \mathrm{N} 100^{\circ} 15^{\prime} 34^{\prime \prime} \mathrm{E}$ & \\
\hline & Phichit-Mueang & $16^{\circ} 26^{\prime} 18^{\prime \prime} \mathrm{N} 100^{\circ} 21^{\prime} \mathrm{O}^{\prime \prime} \mathrm{E}$ & \\
\hline & Sukhothai-Mueang & $17^{\circ} 0^{\prime} 28^{\prime \prime} \mathrm{N} 99^{\circ} 49^{\prime} 23^{\prime \prime} \mathrm{E}$ & \\
\hline & Uttaradit-Mueang* & $17^{\circ} 37^{\prime} 33^{\prime \prime} \mathrm{N} 100^{\circ} 5^{\prime} 48^{\prime \prime} \mathrm{E}$ & \\
\hline & Chiang Mai-Mueang & $18^{\circ} 47^{\prime} 25^{\prime \prime} \mathrm{N} 98^{\circ} 59^{\prime} 4^{\prime \prime} \mathrm{E}$ & \\
\hline & Khon Kean-Mueang & $16^{\circ} 26^{\prime} 18^{\prime \prime} \mathrm{N} 102^{\circ} 50^{\prime} 20^{\prime \prime} \mathrm{E}$ & \\
\hline & Udonthani-Mueang * & $17^{\circ} 24^{\prime} 54^{\prime \prime} \mathrm{N} 102^{\circ} 47^{\prime} 12^{\prime \prime} \mathrm{E}$ & \\
\hline & Rayong-Mueang* & $12^{\circ} 40^{\prime} 6^{\prime \prime} \mathrm{N} 101^{\circ} 16^{\prime} 30^{\prime \prime} \mathrm{E}$ & \\
\hline \multirow[t]{4}{*}{ Lambdacyhalothrin } & Sra Kaeo-Mueang & $13^{\circ} 48^{\prime} 52^{\prime \prime} \mathrm{N} 102^{\circ} 4^{\prime} 20^{\prime \prime} \mathrm{E}$ & Satimai 2010 [100] \\
\hline & Rayong-Mueang & 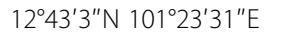 & \\
\hline & Chanthaburi-Mueang & $12^{\circ} 36^{\prime} 40^{\prime \prime} \mathrm{N} 102^{\circ} 6^{\prime} 15^{\prime \prime} \mathrm{E}$ & \\
\hline & Trat-Mueang & $12^{\circ} 13^{\prime} 54^{\prime \prime} \mathrm{N} 102^{\circ} 30^{\prime} 48^{\prime \prime} \mathrm{E}$ & \\
\hline \multirow[t]{12}{*}{ Malathion } & Tak-Mae Sot & $16^{\circ} 46^{\prime} \mathrm{N} 98^{\circ} 34^{\prime} \mathrm{E}$ & Thanispong et al. 2008 [14] \\
\hline & Khon Kean-Mueang & $16^{\circ} 25^{\prime} \mathrm{N} 102^{\circ} 50^{\prime} \mathrm{E}$ & \\
\hline & Surat Thani-Mueang & $9^{\circ} 08^{\prime} \mathrm{N} 99^{\circ} 20^{\prime} \mathrm{E}$ & \\
\hline & Nakhon Sawan-Mueang & $15^{\circ} 42^{\prime} \mathrm{N} 100^{\circ} 08^{\prime} \mathrm{E}$ & \\
\hline & Nonthaburi-Mueang * & $13^{\circ} 51^{\prime} 44^{\prime \prime} \mathrm{N} 100^{\circ} 30^{\prime} 48^{\prime \prime} \mathrm{E}$ & Paeporn et al. 2010 [99] \\
\hline & Sra Kaeo-Mueang & $13^{\circ} 48^{\prime} 53^{\prime \prime} \mathrm{N} 102^{\circ} 4^{\prime} 19^{\prime \prime} \mathrm{E}$ & \\
\hline & Lopburi-Mueang & $14^{\circ} 47^{\prime} 53^{\prime \prime} \mathrm{N} 100^{\circ} 39^{\prime} 13^{\prime \prime} \mathrm{E}$ & \\
\hline & Suphanburi -Mueang * & $14^{\circ} 29^{\prime} 4^{\prime \prime} \mathrm{N} 100^{\circ} 7^{\prime} 25^{\prime \prime} \mathrm{E}$ & \\
\hline & Angthong-Mueang & $14^{\circ} 35^{\prime} 19^{\prime \prime} \mathrm{N} 100^{\circ} 27^{\prime} 12^{\prime \prime} \mathrm{E}$ & \\
\hline & Singburi-Mueang & $14^{\circ} 53^{\prime} 18^{\prime \prime} \mathrm{N} 100^{\circ} 24^{\prime} 17^{\prime \prime} \mathrm{E}$ & \\
\hline & Phichit-Mueang & $16^{\circ} 26^{\prime} 18^{\prime \prime} \mathrm{N} 100^{\circ} 21^{\prime} \mathrm{O}^{\prime \prime} \mathrm{E}$ & \\
\hline & Sukhothai-Mueang * & 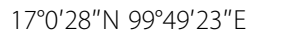 & \\
\hline
\end{tabular}


Table 3 Locations in Thailand with Aedes aegypti populations tested against insecticides using the WHO standard contact assay (2000-2011) (Continued)

\begin{tabular}{|c|c|c|c|}
\hline & Uttaradit-Mueang * & $17^{\circ} 37^{\prime} 33^{\prime \prime} \mathrm{N} 100^{\circ} 5^{\prime} 48^{\prime \prime} \mathrm{E}$ & \\
\hline & Uthaithani-Mueang & $15^{\circ} 22^{\prime} 46^{\prime \prime} \mathrm{N} 100^{\circ} 1^{\prime} 29^{\prime \prime} \mathrm{E}$ & \\
\hline & Khon Kean-Mueang * & 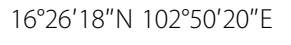 & \\
\hline & Udonthani-Mueang * & $17^{\circ} 24^{\prime} 54^{\prime \prime} \mathrm{N} 102^{\circ} 47^{\prime} 12^{\prime \prime} \mathrm{E}$ & \\
\hline \multirow[t]{40}{*}{ Permethrin } & Chiang Mai- Mae Tang & $19^{\circ} 8^{\prime} \mathrm{N} 98^{\circ} 51^{\prime} \mathrm{E}$ & Prapanthadara et al. 2002 [102] \\
\hline & Chiang Mai-Mueang & $18^{\circ} 46^{\prime} \mathrm{N} 98^{\circ} 57^{\prime} \mathrm{E}$ & Somboon et al. 2003 [93] \\
\hline & Nan-Mueang & $18^{\circ} 47^{\prime} \mathrm{N} 100^{\circ} 43^{\prime} \mathrm{E}$ & \\
\hline & Lampang-Mueang & $19^{\circ} 11^{\prime} \mathrm{N} 98^{\circ} 54^{\prime} \mathrm{E}$ & \\
\hline & Ratchaburi- Pongsawai & 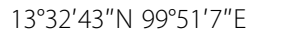 & Paeporn et al. 2004 [34] \\
\hline & Ratchaburi- KhuBua & 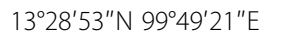 & \\
\hline & Chiang Mai-Mae Tang & $19^{\circ} 9^{\prime} \mathrm{N} 98^{\circ} 47^{\prime} \mathrm{E}$ & Lumjuan et al. 2005 [103] \\
\hline & Tak-Mae Pa & $16^{\circ} 45^{\prime} \mathrm{N} 98^{\circ} 33^{\prime} \mathrm{E}$ & Ponlawat et al. 2005 [98] \\
\hline & Tak-Mae Pa & $16^{\circ} 45^{\prime} \mathrm{N} 98^{\circ} 34^{\prime} \mathrm{E}$ & \\
\hline & Nakhon Sawan-Phayuhakhiri & $15^{\circ} 29^{\prime} \mathrm{N} 100^{\circ} 8^{\prime} \mathrm{E}$ & \\
\hline & Surat Thani-Tha Chana & $9^{\circ} 34^{\prime} \mathrm{N} 99^{\circ} 07^{\prime} \mathrm{E}$ & \\
\hline & Phatthalung-Mueang & $7^{\circ} 30^{\prime} \mathrm{N} 100^{\circ} 03^{\prime} \mathrm{E}$ & \\
\hline & Nakhon Ratchasima-Kham Thale So & $15^{\circ} 05^{\prime} \mathrm{N} 101^{\circ} 54^{\prime} \mathrm{E}$ & \\
\hline & Chiang Mai- Mae Tang & $19^{\circ} 8^{\prime} \mathrm{N} 98^{\circ} 51^{\prime} \mathrm{E}$ & Prapanthadara et al. 2005 [104] \\
\hline & Chiang Mai-Mae Tang & $19^{\circ} 9^{\prime} \mathrm{N} 98^{\circ} 52^{\prime} \mathrm{E}$ & Sathantriphop et al. 2006 [106] \\
\hline & Nonthaburi-Baan Suan & $13^{\circ} 51^{\prime} \mathrm{N} 100^{\circ} 29^{\prime} \mathrm{E}$ & \\
\hline & Bangkok-Bangkoknoi & $13^{\circ} 45^{\prime} 40^{\prime \prime} \mathrm{N} 100^{\circ} 2^{\prime} 1.9^{\prime \prime} \mathrm{E}$ & Jirakanjanakit et al. 2007 [13] \\
\hline & Chonburi-Sriracha & $13^{\circ} 19^{\prime \prime} \mathrm{N} 101^{\circ} 11.8^{\prime \prime} \mathrm{E}$ & \\
\hline & Songkhla-Mueang & $07^{\circ} 1^{\prime} 41.8^{\prime \prime} \mathrm{N} 100^{\circ} 3^{\prime} 54.6^{\prime \prime} \mathrm{E}$ & \\
\hline & Kanchanaburi-Tamaka & $13^{\circ} 55^{\prime} 15^{\prime \prime} \mathrm{N} 99^{\circ} 45^{\prime} 56^{\prime \prime} \mathrm{E}$ & \\
\hline & Bangkok-Hauykwang & $13^{\circ} 4^{\prime} 47.4^{\prime \prime} \mathrm{N} 100^{\circ} 3^{\prime} 52.3^{\prime \prime} \mathrm{E}$ & \\
\hline & Bangkok-Laksi & $13^{\circ} 5^{\prime} 28.2^{\prime \prime} \mathrm{N} 100^{\circ} 3^{\prime} 41.2^{\prime \prime} \mathrm{E}$ & \\
\hline & Bangkok-Ladkrabang & $13^{\circ} 4^{\prime} 47.5^{\prime \prime} \mathrm{N} 100^{\circ} 4^{\prime} 23.6^{\prime \prime} \mathrm{E}$ & \\
\hline & Bangkok-Rasburana & $13^{\circ} 3^{\prime} 59.2^{\prime \prime} \mathrm{N} 100^{\circ} 3^{\prime} 58.8^{\prime \prime} \mathrm{E}$ & \\
\hline & Bangkok-Panusnikom & $13^{\circ} 2^{\prime} 2.9^{\prime \prime} \mathrm{N} 101^{\circ} 1^{\prime} 5.3^{\prime \prime} \mathrm{E}$ & \\
\hline & Bangkok-Banglamung & $12^{\circ} 5^{\prime} 42.2^{\prime \prime} \mathrm{N} 100^{\circ} 5^{\prime} 32.2^{\prime \prime} \mathrm{E}$ & \\
\hline & Nakhon Sawan-Taklee & $15^{\circ} 1^{\prime} 53^{\prime \prime} \mathrm{N} 100^{\circ} 1^{\prime} 48.2^{\prime \prime} \mathrm{E}$ & \\
\hline & Nakhon Sawan-Muang & $15^{\circ} 4^{\prime} 47^{\prime \prime} \mathrm{N} 100^{\circ} 34.7^{\prime \prime} \mathrm{E}$ & \\
\hline & Nakhon Sawan-Krok Pra & $15^{\circ} 3^{\prime} 12^{\prime \prime} \mathrm{N} 100^{\circ} 33.7^{\prime \prime} \mathrm{E}$ & \\
\hline & Nakhon Ratchasima-Senagsang & $14^{\circ} 2^{\prime} 35.2^{\prime \prime} \mathrm{N} 102^{\circ} 2^{\prime} 45.6^{\prime \prime} \mathrm{E}$ & \\
\hline & Nakhon Sawan-Mae Pern & $15^{\circ} 3^{\prime} 21^{\prime \prime} \mathrm{N} 99^{\circ} 2^{\prime} 48^{\prime \prime} \mathrm{E}$ & \\
\hline & Nakhon Sawan-Mae Wong & $15^{\circ} 4^{\prime} 35^{\prime \prime} \mathrm{N} 99^{\circ} 3^{\prime} 7.4^{\prime \prime} \mathrm{E}$ & \\
\hline & Bangkok-Hauykwang & $13^{\circ} 45^{\prime} 54^{\prime \prime} \mathrm{N} 100^{\circ} 34^{\prime} 39^{\prime \prime}$ & Pethuan et al. 2007 [108] \\
\hline & Bangkok-Laksi & 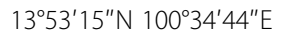 & \\
\hline & Chonburi-Panusnikom & $13^{\circ} 27^{\prime} 6^{\prime \prime} \mathrm{N} 101^{\circ} 10^{\prime} 36^{\prime \prime} \mathrm{E}$ & \\
\hline & Chonburi-Banglamung & $12^{\circ} 58^{\prime} 36^{\prime \prime} \mathrm{N} 100^{\circ} 54^{\prime} 48^{\prime \prime} \mathrm{E}$ & \\
\hline & Nakhon Sawan & $15^{\circ} 15^{\prime} 47^{\prime \prime} \mathrm{N} 100^{\circ} 20^{\prime} 37^{\prime \prime} \mathrm{E}$ & \\
\hline & Nakhon Sawan-Mueang & $15^{\circ} 44^{\prime} 15^{\prime \prime} \mathrm{N} 100^{\circ} 5^{\prime} 21^{\prime \prime} \mathrm{E}$ & \\
\hline & Nakhon Sawan-Krok Pra & $15^{\circ} 34^{\prime} 48^{\prime \prime} \mathrm{N} 100^{\circ} 0^{\prime} 13^{\prime \prime} \mathrm{E}$ & \\
\hline & Nakhon Ratchasrima-Serngsang & $14^{\circ} 25^{\prime} 34^{\prime \prime} \mathrm{N} 102^{\circ} 27^{\prime} 38^{\prime \prime} \mathrm{E}$ & \\
\hline
\end{tabular}


Table 3 Locations in Thailand with Aedes aegypti populations tested against insecticides using the WHO standard contact assay (2000-2011) (Continued)

\begin{tabular}{|c|}
\hline Nakhon Sawan-Mae Pern \\
\hline Nakhon Sawan-Mae Wc \\
\hline Chonburi \\
\hline Phang Nga \\
\hline Phang Nga-Thap Pud \\
\hline Chiang Mai-Mueang \\
\hline Kanchanaburi-Sai Yok \\
\hline Nonthaburi-Mueang \\
\hline Songkhla-Mueang \\
\hline Satun-Mueang \\
\hline Bangkok-Chatuchak \\
\hline Lampang-Mueang \\
\hline Tak-Mae Sot \\
\hline Khon Kean-Mueang \\
\hline Surat Thani-Mueang \\
\hline Nakhon Sawan-Mueang \\
\hline Nonthaburi-Mueang \\
\hline Nakhon Pathom-Mueang \\
\hline Sra Kaeo-Mueang \\
\hline Saraburi-Mueang \\
\hline Lopburi-Mueang \\
\hline Suphanburi-Mueang \\
\hline Angthong-Mueang \\
\hline Singburi-Mueang \\
\hline Kanchanaburi-Mueang \\
\hline Phitsanulok-Mueang \\
\hline Phichit-Mueang \\
\hline Sukhothai-Mueang \\
\hline Uttaradit-Mueang \\
\hline Lamphun-Mueang \\
\hline Uthaithani-Mueang \\
\hline Chiang Mai-Mueang \\
\hline Chiang Rai-Mueang \\
\hline Khon Kean-Mueang \\
\hline Udonthani-Mueang \\
\hline Chai yaphum-Mueang \\
\hline Nongkhai-Mueang \\
\hline Nakhon Nayok-Mueang \\
\hline Rayong-Mueang \\
\hline Prachinburi-Mueang \\
\hline Chonburi-Mueang \\
\hline Sra Kaeo-Mueang \\
\hline Rayong-Mueang \\
\hline Chanthaburi-Mueang \\
\hline
\end{tabular}

$15^{\circ} 39^{\prime} 28^{\prime \prime} \mathrm{N} 99^{\circ} 28^{\prime} 9^{\prime \prime} \mathrm{E}$

$15^{\circ} 46^{\prime} 52^{\prime \prime} \mathrm{N} 99^{\circ} 31^{\prime} 9^{\prime \prime} \mathrm{E}$

$13^{\circ} 22^{\prime} 0^{\prime \prime} \mathrm{N} 100^{\circ} 58^{\prime} 60^{\prime \prime} \mathrm{E}$

Rajatileka et al. 2008 [107]

$8^{\circ} 28^{\prime} 0^{\prime \prime} \mathrm{N} 98^{\circ} 31^{\prime} 60^{\prime \prime} \mathrm{E}$

$8^{\circ} 31^{\prime} 0^{\prime \prime} \mathrm{N} 98^{\circ} 37^{\prime} 60^{\prime \prime} \mathrm{E}$

$18^{\circ} 47^{\prime} \mathrm{N} 99^{\circ} 00^{\prime} \mathrm{E}$

Thanispong et al. 2008 [14]

$14^{\circ} 20^{\prime} \mathrm{N} 98^{\circ} 59^{\prime} \mathrm{E}$

$13^{\circ} 53^{\prime} \mathrm{N} 100^{\circ} 29^{\prime} \mathrm{E}$

$7^{\circ} 11^{\prime} \mathrm{N} 100^{\circ} 35^{\prime} \mathrm{E}$

$6^{\circ} 37^{\prime} \mathrm{N} 100^{\circ} 03^{\prime} \mathrm{E}$

$13^{\circ} 50^{\prime} \mathrm{N} 100^{\circ} 34^{\prime} \mathrm{E}$

$18^{\circ} 17^{\prime} \mathrm{N} 99^{\circ} 29^{\prime} \mathrm{E}$

$16^{\circ} 46^{\prime} \mathrm{N} 98^{\circ} 34^{\prime} \mathrm{E}$

$16^{\circ} 25^{\prime} \mathrm{N} 102^{\circ} 50^{\prime} \mathrm{E}$

$9^{\circ} 08^{\prime} \mathrm{N} 99^{\circ} 20^{\prime} \mathrm{E}$

$15^{\circ} 42^{\prime} \mathrm{N} 100^{\circ} 08^{\prime} \mathrm{E}$

$13^{\circ} 51^{\prime} 44^{\prime \prime} \mathrm{N} \mathrm{100} 30^{\prime} 48^{\prime \prime} \mathrm{E}$

Paeporn et al. 2010 [99]

$13^{\circ} 49^{\prime} 11^{\prime \prime N} 100^{\circ} 3^{\prime} 57^{\prime \prime}$

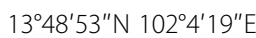

$14^{\circ} 31^{\prime} 38^{\prime \prime} \mathrm{N} \mathrm{100} 54^{\prime} 35^{\prime \prime} \mathrm{E}$

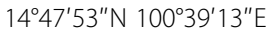

$14^{\circ} 29^{\prime} 4^{\prime \prime} \mathrm{N} 100^{\circ} 7^{\prime} 25^{\prime \prime} \mathrm{E}$

$14^{\circ} 35^{\prime} 19^{\prime \prime} \mathrm{N} 100^{\circ} 27^{\prime} 12^{\prime \prime} \mathrm{E}$

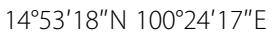

$14^{\circ} 0^{\prime} 12^{\prime \prime} \mathrm{N} 99^{\circ} 33^{\prime} \mathrm{O}^{\prime \prime} \mathrm{E}$

$16^{\circ} 49^{\prime} 29^{\prime \prime} \mathrm{N} 100^{\circ} 15^{\prime} 34^{\prime \prime} \mathrm{E}$

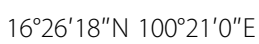

$17^{\circ} 0^{\prime} 28^{\prime \prime} \mathrm{N} 99^{\circ} 49^{\prime} 23^{\prime \prime} \mathrm{E}$

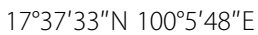

$18^{\circ} 34^{\prime} 42^{\prime \prime} \mathrm{N} 99^{\circ} 1^{\prime} 6^{\prime \prime} \mathrm{E}$

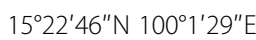

$18^{\circ} 47^{\prime} 25^{\prime \prime} \mathrm{N} 98^{\circ} 59^{\prime} 4^{\prime \prime} \mathrm{E}$

$19^{\circ} 54^{\prime} 31^{\prime \prime N}$ 9949'57"E

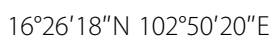

$17^{\circ} 24^{\prime} 54^{\prime \prime} \mathrm{N} 102^{\circ} 47^{\prime} 12^{\prime \prime} \mathrm{E}$

$15^{\circ} 48^{\prime} 35^{\prime \prime} \mathrm{N} 102^{\circ} 1^{\prime} 13^{\prime \prime} \mathrm{E}$

$17^{\circ} 52^{\prime} 48^{\prime \prime} \mathrm{N} 102^{\circ} 44^{\prime} 30^{\prime \prime} \mathrm{E}$

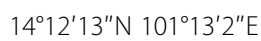

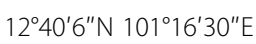

$14^{\circ} 3^{\prime} 2^{\prime \prime} \mathrm{N} 101^{\circ} 22^{\prime} \mathrm{O}^{\prime \prime} \mathrm{E}$

$13^{\circ} 21^{\prime} 43^{\prime \prime} \mathrm{N} 100^{\circ} 58^{\prime} 45^{\prime \prime} \mathrm{E}$

$13^{\circ} 48^{\prime} 52^{\prime \prime} \mathrm{N} 102^{\circ} 4^{\prime} 20^{\prime \prime} \mathrm{E}$

Satimai 2010 [100]

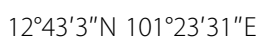

$12^{\circ} 36^{\prime} 40^{\prime \prime} \mathrm{N} 102^{\circ} 6^{\prime} 15^{\prime \prime} \mathrm{E}$ 
Table 3 Locations in Thailand with Aedes aegypti populations tested against insecticides using the WHO standard contact assay (2000-2011) (Continued)

\begin{tabular}{|c|c|c|c|}
\hline & Trat-Mueang & $12^{\circ} 13^{\prime} 54^{\prime \prime} \mathrm{N} 102^{\circ} 30^{\prime} 48^{\prime \prime} \mathrm{E}$ & \\
\hline & Bangkok-Kannayaw & $13^{\circ} 50^{\prime} \mathrm{N} 100^{\circ} 40^{\prime} \mathrm{E}$ & Chuaycharoensuk et al. 2011[35] \\
\hline & Chanthaburi-Mueang & $12^{\circ} 39^{\prime} \mathrm{N} 102^{\circ} 7^{\prime} \mathrm{E}$ & \\
\hline & Chonburi-Mueang & $13^{\circ} 19^{\prime} \mathrm{N} 100^{\circ} 55^{\prime} \mathrm{E}$ & \\
\hline & Prachinburi-Mueang & $14^{\circ} 7^{\prime} \mathrm{N} 101^{\circ} 21^{\prime} \mathrm{E}$ & \\
\hline & Buri Rum-Lam Plai Mat & $15^{\circ} 00^{\prime} \mathrm{N} 102^{\circ} 50^{\prime} \mathrm{E}$ & \\
\hline & Kalasin-Huaypung & $16^{\circ} 40^{\prime} \mathrm{N} 103^{\circ} 53^{\prime} \mathrm{E}$ & \\
\hline & Prachinburi-Mueang & $14^{\circ} 6^{\prime} \mathrm{N} 101^{\circ} 19^{\prime} \mathrm{E}$ & \\
\hline & Khon Kean-Mueang & $16^{\circ} 19^{\prime} \mathrm{N} 102^{\circ} 47^{\prime} \mathrm{E}$ & \\
\hline & Nakhon Ratchasima-WangNam Kheow & $14^{\circ} 26^{\prime} \mathrm{N} 101^{\circ} 47^{\prime} \mathrm{E}$ & \\
\hline & Srisaket-Uthumpronpisai & $15^{\circ} 08^{\prime} \mathrm{N} 104^{\circ} 12^{\prime} \mathrm{E}$ & \\
\hline & Surin-Dontoom & $15^{\circ} 14^{\prime} \mathrm{N} 103^{\circ} 30^{\prime} \mathrm{E}$ & \\
\hline & Roi Et-Suwannaphum & $15^{\circ} 40^{\prime} \mathrm{N} 103^{\circ} 49^{\prime} \mathrm{E}$ & \\
\hline & Nakhon Ratchasima-WangNam Kheow & $14^{\circ} 24^{\prime} \mathrm{N} 101^{\circ} 51^{\prime} \mathrm{E}$ & \\
\hline & Udonthani-Wungsammor & $17^{\circ} 3^{\prime} \mathrm{N} 103^{\circ} 26^{\prime} \mathrm{E}$ & \\
\hline & Chiang Mai-Mueang & $18^{\circ} 46^{\prime} \mathrm{N} 98^{\circ} 56^{\prime} \mathrm{E}$ & \\
\hline & Chiang Rai-Mae Chun & $20^{\circ} 08^{\prime} \mathrm{N} 99^{\circ} 51^{\prime} \mathrm{E}$ & \\
\hline & Kampaeng Phet-Kanuworralukburi & $16^{\circ} 00^{\prime} \mathrm{N} 99^{\circ} 48^{\prime} \mathrm{E}$ & \\
\hline & Lampang-Mueang & $18^{\circ} 14^{\prime} \mathrm{N} 99^{\circ} 26^{\prime} \mathrm{E}$ & \\
\hline & Lamphun-Mueang & $18^{\circ} 37^{\prime} \mathrm{N} 99^{\circ} 00^{\prime} \mathrm{E}$ & \\
\hline & Nakhon Sawan-Mueang & $15^{\circ} 40^{\prime} \mathrm{N} 100^{\circ} 05^{\prime} \mathrm{E}$ & \\
\hline & Phrae-Mueang & $18^{\circ} 05^{\prime} \mathrm{N} 100^{\circ} 12^{\prime} \mathrm{E}$ & \\
\hline & Tak-Mae Sot & $16^{\circ} 43^{\prime} \mathrm{N} 98^{\circ} 34^{\prime} \mathrm{E}$ & \\
\hline & Uthaithani-Ban Rai & $15^{\circ} 12^{\prime} \mathrm{N} 99^{\circ} 41^{\prime} \mathrm{E}$ & \\
\hline & Chumphon-Mueang & $10^{\circ} 30^{\prime} \mathrm{N} 99^{\circ} 07^{\prime} \mathrm{E}$ & \\
\hline & Phang Nga-Takuaytung & $08^{\circ} 12^{\prime} \mathrm{N} 98^{\circ} 17^{\prime} \mathrm{E}$ & \\
\hline & Phatthalung-Pa Bon & $07^{\circ} 16^{\prime} \mathrm{N} 100^{\circ} 09^{\prime} \mathrm{E}$ & \\
\hline & Phuket-Mueang & $07^{\circ} 53^{\prime} \mathrm{N} 98^{\circ} 23^{\prime} \mathrm{E}$ & \\
\hline & Prachuap Khiri Khan-Hua Hin & $12^{\circ} 33^{\prime} \mathrm{N} 99^{\circ} 53^{\prime} \mathrm{E}$ & \\
\hline & Songkhla-Namom & $06^{\circ} 54^{\prime} \mathrm{N} 100^{\circ} 32^{\prime} \mathrm{E}$ & \\
\hline & Songkhla-Ranode & $07^{\circ} 52^{\prime} \mathrm{N} 100^{\circ} 18^{\prime} \mathrm{E}$ & \\
\hline & Songkhla-Sadao & $06^{\circ} 45^{\prime} \mathrm{N} 100^{\circ} 24^{\prime} \mathrm{E}$ & \\
\hline & Songkhla-Had Yai & $07^{\circ} 00^{\prime} \mathrm{N} 100^{\circ} 27^{\prime} \mathrm{E}$ & \\
\hline & Surat Thani-Mueang & $09^{\circ} 02^{\prime} \mathrm{N} 99^{\circ} 22^{\prime} \mathrm{E}$ & \\
\hline \multirow[t]{4}{*}{ Propoxur } & Nakhon Sawan-Mae Wong & 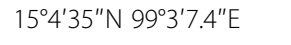 & Jirakanjanakit et al. 2007 [13] \\
\hline & Nakhon Sawan-Mae Wong & $15^{\circ} 46^{\prime} 52^{\prime \prime} \mathrm{N} 99^{\circ} 31^{\prime} 9^{\prime \prime} \mathrm{E}$ & Pethuan et al. 2007 [108] \\
\hline & Surat Thani-Mueang & $9^{\circ} 08^{\prime} \mathrm{N} 99^{\circ} 20^{\prime} \mathrm{E}$ & Thanispong et al. 2008 [14] \\
\hline & Nakhon Sawan-Muang & $15^{\circ} 42^{\prime} \mathrm{N} 100^{\circ} 08^{\prime} \mathrm{E}$ & \\
\hline \multirow[t]{6}{*}{ Temephos } & Tak-Mae Pa & $16^{\circ} 45^{\prime} \mathrm{N} 98^{\circ} 33^{\prime} \mathrm{E}$ & Ponlawat et al. 2005 [98] \\
\hline & Tak-Mae Pa & $16^{\circ} 45^{\prime} \mathrm{N} 98^{\circ} 34^{\prime} \mathrm{E}$ & \\
\hline & Nakhon Sawan-Phayuhakhiri & $15^{\circ} 29^{\prime} \mathrm{N} 100^{\circ} 8^{\prime} \mathrm{E}$ & \\
\hline & Surat Thani-Tha Chana & $9^{\circ} 34^{\prime} \mathrm{N} 99^{\circ} 07^{\prime} \mathrm{E}$ & \\
\hline & Phatthalung-Mueang & $7^{\circ} 30^{\prime} \mathrm{N} 100^{\circ} 03^{\prime} \mathrm{E}$ & \\
\hline & Roi Et-Mueang & $16^{\circ} 03^{\prime} \mathrm{N} 103^{\circ} 39^{\prime} \mathrm{E}$ & Saelim et al. 2005 [110] \\
\hline
\end{tabular}




\section{Table 3 Locations in Thailand with Aedes aegypti populations tested against insecticides using the WHO standard contact assay (2000-2011) (Continued)}

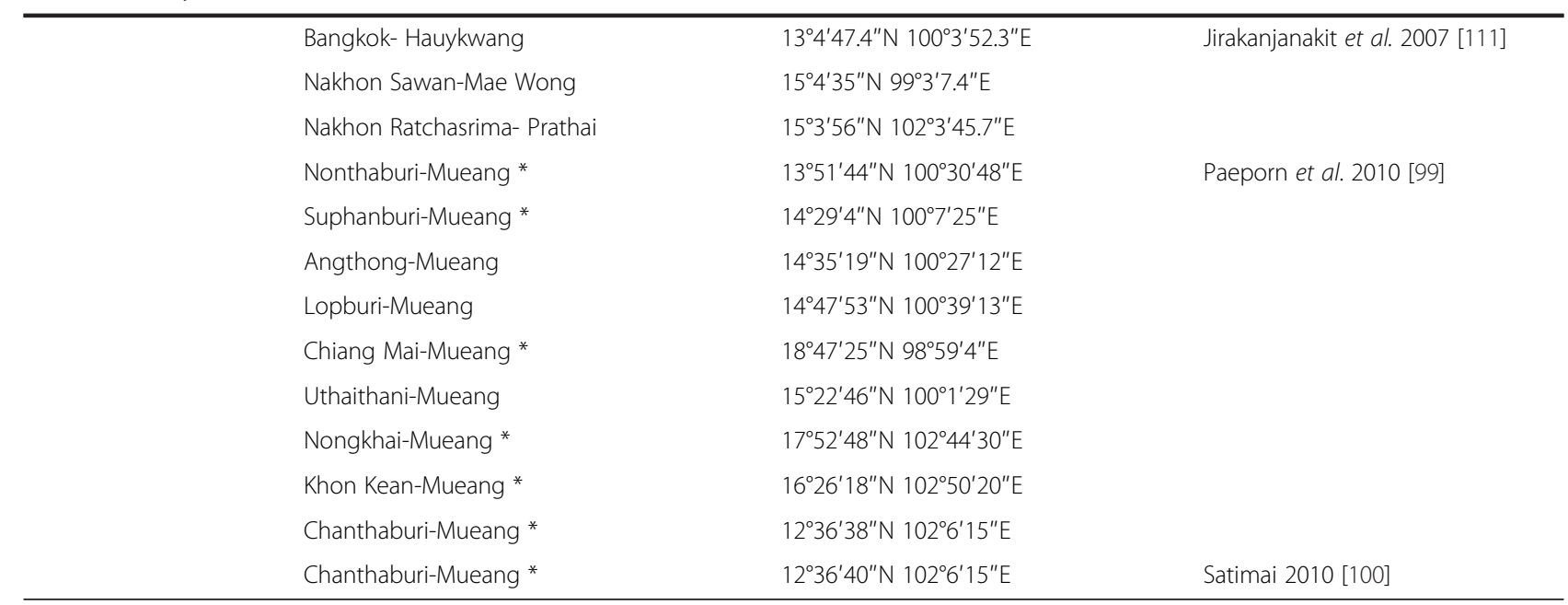

*Evidence of low-grade insecticide resistance.

circulating microfilaria in the peripheral blood occurring between 18.00 and $20.00 \mathrm{~h}[59,60]$.

\section{Malaria vectors}

Of the approximately 73 Anopheles species found in Thailand, select members of the Leucosphyrus Group (Neomyzomyia Series), Maculatus Group (Neocellia Series), and Minimus Subgroup (Myzomyia Series), as the most important malaria vectors in the country [38]. Five species within these 3 assemblages are incriminated as primary malaria vectors in Thailand, including Anopheles baimaii (previously An. dirus D) [61], Anopheles dirus [61,62], Anopheles minimus (previously species A) [63], Anopheles pseudowillmori [61] and Anopheles aconitus [61,64,65]. Manguin et al. [38] provides a current review of the vectorial capacity and bionomics of malaria vectors in the SEA region.

Several other potential vectors of malaria in Thailand that have a close association with humans, included $A n$. maculatus, Anopheles epiroticus (= An. sundaicus A), Anopheles karwari, Anopheles philippinensis, and Anopheles tessellatus. Additionally, some members of the Barbirostris Group, subgenus Anopheles (Anopheles barbirostris and Anopheles campestris) exhibit malaria vector potential in Thailand [66].

\section{Dengue vectors}

Only two species of Aedes mosquitoes, Aedes aegypti and Aedes albopictus are considered as primary vectors of dengue viruses in Thailand [67]. Aedes aegypti is highly anthropophilic and often propagates in and around human dwellings with a high propensity for resting inside houses. Larval habitats are typically artificial containers holding fresh water such as discarded tires, flower pots, drums, refuse bottles and cans, and other water storage devices [67,68]. Aedes albopictus is believed to be a native to Southeast Asia [69]. Aedes albopictus prefers to breed in natural habitats like tree holes, bamboo stumps and other natural containers but will also utilize outdoor man-made habitats that typically contain a higher amount of organic matter than tolerated by Ae. aegypti. Predominantly a 'rural' species in Thailand, Ae. albopictus has been reported invading residential areas of larger urban zones, especially in the vicinity of Bangkok [70].

Female Ae. albopictus also displays a greater preference to rest and feed outdoors [71,72]. Similar to $A e$. aegypti, it is a daytime feeder and can be found resting in shady areas in shrubs nearer ground level [73]. Likewise, Ae. albopictus blood feeding activity peaks in the early morning and late afternoon and is considered an aggressive biter with a wider host feeding range (human, domestic and wild animals) than Ae. aegypti [74]. This species is also known to be a competent vector of several other viral human pathogens, including Chikungunya virus [75,76] and Eastern equine encephalitis virus [77].

\section{Lymphatic filariasis vectors}

In Asia, at least 36 mosquito species belonging to six genera have been incriminated as either primary or secondary vectors of $W$. bancrofti, with the majority being Anopheles species (24) followed by aedine mosquitoes (7 spp.), Culex (4 spp.) and two Mansonia (M. dives, M. uniformis) [38]. In Thailand, two species of Mansonia and five Anopheles are vectors of Brugia malayi [78]. Larval habitats for many of these vector species are commonly marshes/swamps found in close proximity to villages. The NSP form of the parasite has been experimentally and naturally transmitted by several mosquito genera, including Aedes, Mansonia, Anopheles and Downsiomyia [58,60]. In Thailand, lymphatic 
filariasis and malaria parasites can naturally share the same vector species, in particular Anopheles dirus and Anopheles minimus complexes, the Anopheles maculatus group, Anopheles aconitus and Anopheles vagus [38]. Active cases of filariasis in Thailand have declined over the past 20 years. Transmission is now found primarily with at-risk Thai migrants who enter endemic areas where vectors are common, especially along the Thai-Myanmar border. Increases in temporary migrant workers along the border have been associated with increases in disease transmission in the region. The prevalence of filariasis among a group of migrant workers in Tak Province, western Thailand, was approximately $4.4 \%$ and $2.4 \%$ in Prachuab Kiri Khan Province, southern Thailand $[79,80]$.

\section{Japanese encephalitis vectors}

In Thailand, JE virus is maintained enzootically within the rice field-breeding mosquitoes $C x$. tritaeniorhynchus, $C x$. fuscocephala, and Cx. gelidus [81]. Burke and Leake [82] have also reported $C x$. pseudovishnui and $C x$. vishnui to be competent vectors of JE and more recently, the virus has also been isolated from Culex quinquefasciatus in Thailand [83]. These mosquitoes can serve as the 'bridging' vectors to humans from pigs that are the primary amplifying hosts in endemic areas and native wading birds associated with rice fields and natural wetlands as the natural virus reservoirs [84].

\section{Chikungunya vectors}

The virus can be transmitted to humans via several species of mosquitoes, most notably several Aedes species, but also Culex annulirostris, Mansonia uniformis and a few species of Anopheles [85-87]. In Asia, the same two species that transmit dengue viruses, Ae aegypti and Ae. albopictus, are of prime importance in chikungunya transmission in both urban and rural settings $[88,89]$. Aedes albopictus has been shown to have a higher susceptibility and a greater propensity to transmit the chikungunya virus than Ae. aegypti $[75,76,90,91]$. Similar to Ae. aegypti, the normal flight range of Ae. albopictus is generally limited within a radius of 400-600 m from their original larval habitat [92]. Like dengue, the virus has also been shown to be vertically transmitted from an infected female mosquito to her eggs and prodigy. The rapid extension of strains of Ae albopictus worldwide, especially in temperate areas, represents a serious potential threat of chikungunya transmission in areas where it has not been seen before.

\section{Physiological resistance to insecticides}

One of the primary methods of preventing vector-borne disease transmission is to disrupt human-vector contact using chemical means $[12,13]$. Synthetic chemicals of various classes have been used for many years in national public health vector control programs $[1,2]$,

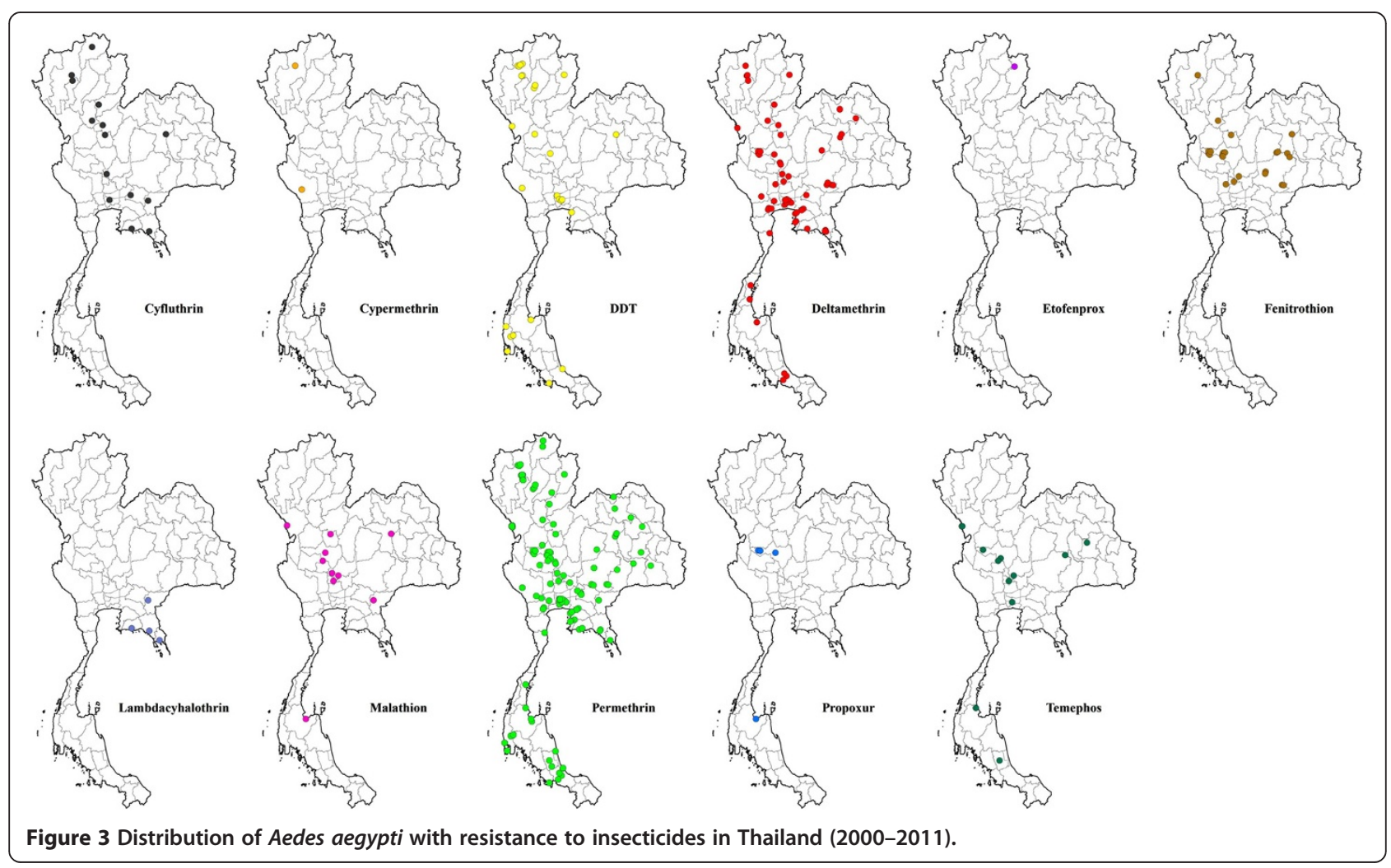




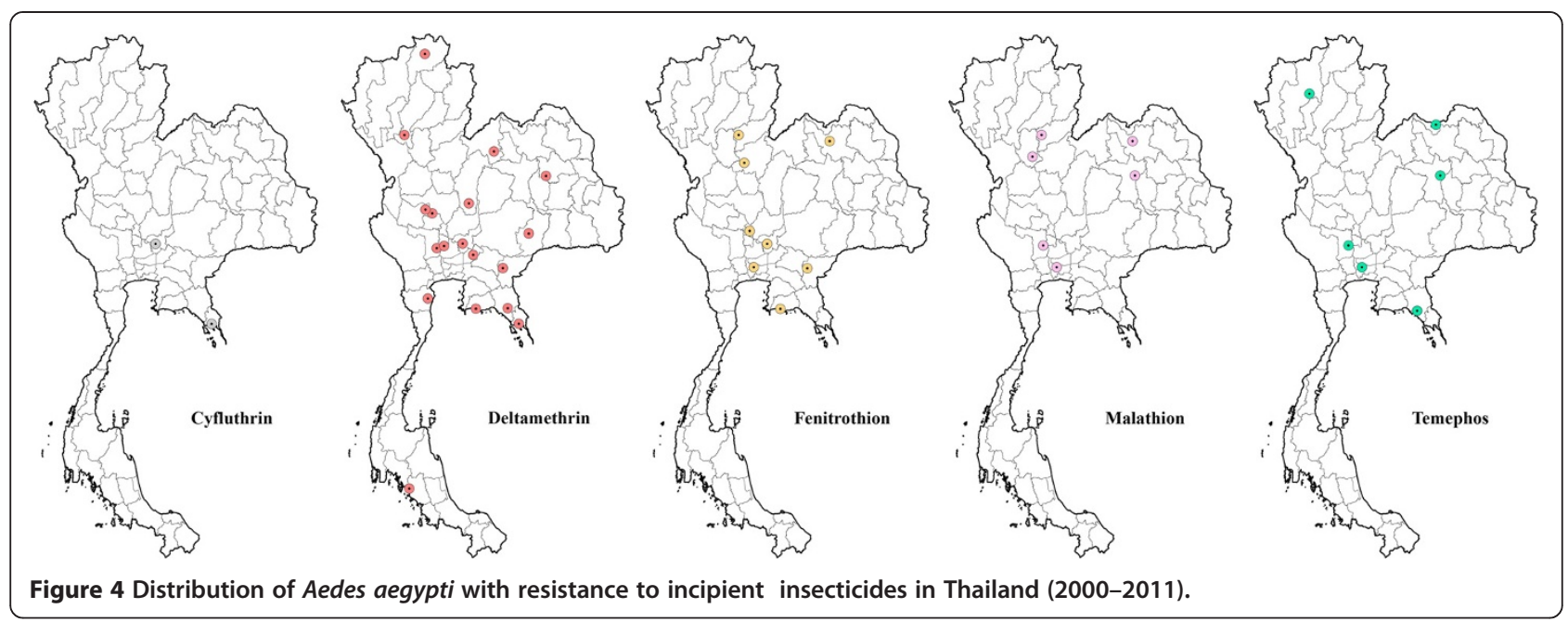

currently the majority of which are pyrethroid-based formulations. In Thailand, combinations of different pyrethroids are commercially available to home owners to control mosquitoes and other indoor/outdoor arthropod pests. Pyrethroids have become relatively inexpensive, provide quick knockdown and are relatively safe compounds to use near humans to control common housefrequenting mosquitoes [8,93]. Since 1994, deltamethrin has been intensively used in organized vector control programs in Thailand to interrupt dengue transmission, generally in response to an outbreak. Numerous pyrethroid-based formulations (e.g., aerosols, coils, spray and gels), that include one or more of 12 different active ingredients, are available commercially to the general public $[8,35,94]$. A survey of 23 household products in public markets in metropolitan Bangkok found 11 containing varying low concentrations of permethrin, 11 with tetramethrin, 4 with bioallethrin and prallethrin, many with a synergist (piperonyl butoxide) added. Only three products had a mixture of deltamethrin (Table 1). One carbamate (propoxur) and one organophosphate (dichlorvos) are also available as an approved mixture in household pest control products. Frequent exposure of mosquito populations to sub-lethal concentrations of these chemicals may result in, or contribute to, the development of insecticide resistance with a direct operational impact on the effective management and prevention of vector-borne diseases [95].

In Thailand, resistance to DDT has been documented in only 2 species of anophelines (Anopheles annularis and Anopheles minimus) (Table 2), located in the northwestern part of the country [96]. In spite of decades of organized control activities using chemicals against malaria vectors, particularly IRS, there has been no published data reporting resistance to any other chemical class or active ingredient (mainly deltamethrin) used for vector control. This interesting finding contrasts significantly with the resistance patterns seen in Ae. aegypti, $A e$ albopictus and $C x$. quinquefasciatus in the country and could be explained by the predominant exophilic/ exophagic behavior of major malaria vectors in the region that would limit the exposure time of mosquitoes with residual insecticides present inside houses (e.g., IRS and bednets).

Table 4 Locations in Thailand with Aedes albopictus populations tested against insecticides using the WHO standard contact assay (2000-2011)

\begin{tabular}{llll}
\hline Insecticide & Location (province-district) & Geographic coordinates (DMS) & Published sources \\
\hline DDT & Chiang Mai-Mae Tang & $19^{\circ} 11^{\prime} \mathrm{N} 98^{\circ} 54^{\prime} \mathrm{E}$ & Somboon et al. 2003 [93] \\
Permethrin & Phatthalung-Mueang & $7^{\circ} 30^{\prime} \mathrm{N} 100^{\circ} 03^{\prime} \mathrm{E}$ & Ponlawat et al. 2005 [98] \\
& Tak-Mae Sot & $16^{\circ} 45^{\prime} \mathrm{N} 98^{\circ} 33^{\prime} \mathrm{E}$ & $16^{\circ} 45^{\prime} \mathrm{N} 98^{\circ} 34^{\prime} \mathrm{E}$ \\
& Tak-Mae Sot & $10^{\circ} 30^{\prime} \mathrm{N} 99^{\circ} 07^{\prime} \mathrm{E}$ & Chuaycharoensuk et al. 2011 [35] \\
& Chumphon-Mueang & $12^{\circ} 33^{\prime} \mathrm{N} 99^{\circ} 53^{\prime} \mathrm{E}$ & \\
& Prachuap Khiri Khan-Hua Hin & $6^{\circ} 54^{\prime} \mathrm{N} 100^{\circ} 32^{\prime} \mathrm{E}$ & \\
& Songkhla-Namom & $6^{\circ} 45^{\prime} \mathrm{N} 100^{\circ} 24^{\prime} \mathrm{E}$ & \\
& Songkhla-Sadao & $9^{\circ} 02^{\prime} \mathrm{N} 99^{\circ} 22^{\prime} \mathrm{E}$ & \\
\hline
\end{tabular}


Resistance or incipient (tolerance) resistance to temephos, a common organophosphate used to control mosquito larvae, has been observed in Ae. aegypti in Thailand $[13,97,98]$. Of 19 populations tested, 12 were found resistant to temephos while 7 populations demonstrated tolerance. The degree of susceptibility to temephos also appeared to vary depending upon the history of chemical usage/exposure in the area. Data on the susceptibility to malathion and fenitrothion (organophosphates) and propoxur (carbamate) in adult Ae. aegypti has been compiled from 2000-2011 based on WHO susceptibility tests (Table 3). The degree of susceptibility to these two chemical groups also varied according to the geographic settings and previous chemical exposure. For example, strong resistance to malathion and fenitrothion in $A e$. aegypti has been reported in the central, northern and northeastern areas of Thailand; whereas only moderate to low grades of resistance have been seen in the far north of the country [13,97]. Only one locality in southern Thailand has reported significant malathion resistance in Ae. aegypti populations [14].

Thanispong et al. [14], reported Ae. aegypti resistant to six different synthetic pyrethroids, namely (cyfluthrin, cypermethrin, deltamethrin, etofenprox, lambda-cyhalothrin, and permethrin) (Table 3). Permethrin resistance is widely distributed in the country, while deltamethrin, lambdacyhalothrin and cyfluthrin resistance has so far shown a much narrower spatial distribution (Figure 3). DDT resistance has been found throughout the country, whereas fenitrothion (OP) was restricted to the central and northern regions of Thailand (Figure 3). In addition, incipient resistance has been reported for three major groups of insecticides, with the majority being synthetic pyrethroids, especially permethrin and deltamethrin (Figure 4). Although data is limited, temephos resistance (OP) in Ae aegypti appears more prevalent than resistance to propoxur (carbamate) (Figure 3). For Aedes albopictus, few reports on physiological resistance patterns are available; however, most of the Ae. albopictus populations sampled have demonstrated resistance to permethrin and one population showed resistance to DDT (Table 4 and Figure 5).

More recent data on pyrethroid resistance in $A e$. aegypti and Ae. albopictus populations from Thailand have been restricted in specific geographical areas $[8,13,14,34,105,106,112]$. Physiological resistance to three different pyrethroids among $32 \mathrm{Ae}$. aegypti strains collected throughout Thailand and five strains of $A e$. albopictus from southern Thailand were investigated [35]. The frequency of resistance to permethrin in Ae. aegypti populations varied between $4 \%$ and $56.4 \%$. All 32 strains of Ae. aegypti were found to have evidence of incipient resistance $(62.5 \%)$ or levels of permethrin postexposure survival deemed clearly resistant (37.5\%). However, the majority of Ae. aegypti strains were found to be

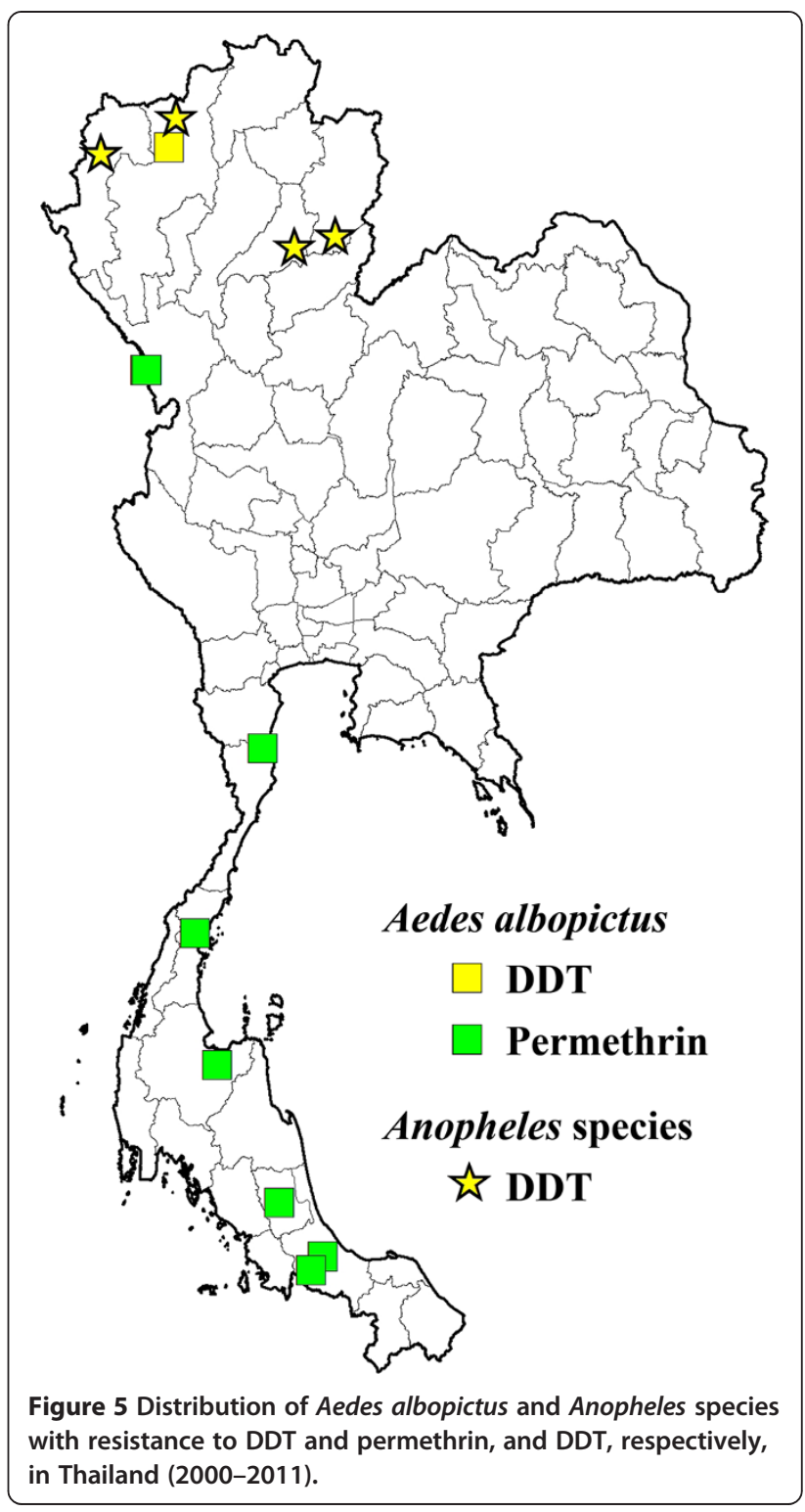

susceptible (> 98\% mortality) to deltamethrin. Four strains of Ae. albopictus showed evidence of incipient resistance to lambda-cyhalothrin and one showed high resistance to permethrin [35].

In $C x$. quinquefasciatus, there were high levels of resistance to DDT, permethrin, deltamethrin and propoxur, whereas susceptibility to malathion and fenitrothion (Table 5, Figure 6) was maintained [93,113]. However, the limited number of sites and populations tested render it difficult to estimate the country-wide importance and impact of insecticide resistance in this mosquito species.

\section{Behavioral responses to insecticides}

Behavioral responses of mosquitoes to insecticides can be observed using various laboratory devices and field 'excito-repellency' assay systems. For laboratory assays, 
Table 5 Locations in Thailand with Culex quinquefasciatus populations tested against insecticides using the WHO standard contact assay (2000-2011)

\begin{tabular}{|c|c|c|c|}
\hline Insecticide & Location (province-district) & Geographic coordinates (DMS) & Sources \\
\hline \multirow[t]{9}{*}{$\overline{\mathrm{DDT}}$} & Chiang Mai- Mueang & $18^{\circ} 47^{\prime} \mathrm{N} 99^{\circ} 1^{\prime} \mathrm{E}$ & Prapanthadara et al. 2000 [96] \\
\hline & Chiang Mai-Mueang & $18^{\circ} 46^{\prime} \mathrm{N} 98^{\circ} 57^{\prime} \mathrm{E}$ & Somboon et al. 2003 [93] \\
\hline & Chiang Mai-San Kampaeng & $18^{\circ} 44^{\prime} 43^{\prime \prime} \mathrm{N} 99^{\circ} 7^{\prime} 13^{\prime \prime} \mathrm{E}$ & \\
\hline & Lampang-Mueang & $18^{\circ} 17^{\prime} 31^{\prime \prime} \mathrm{N} 99^{\circ} 30^{\prime} 16^{\prime \prime} \mathrm{E}$ & \\
\hline & Nan-Tha Wang Pha & $19^{\circ} 7^{\prime} 35^{\prime \prime} \mathrm{N} 100^{\circ} 48^{\prime} 53^{\prime \prime} \mathrm{E}$ & \\
\hline & Nonthaburi-Baan Suan & $13^{\circ} 51^{\prime} \mathrm{N} 100^{\circ} 29^{\prime} \mathrm{E}$ & Sathantriphop et al. 2006 [113] \\
\hline & Bangkok-Phom Pabsatupai & $13^{\circ} 44^{\prime} \mathrm{N} 100^{\circ} 29^{\prime} \mathrm{E}$ & Thanispong et al. 2008 [14] \\
\hline & Nonthaburi-Mueang & $13^{\circ} 50^{\prime} \mathrm{N} 100^{\circ} 31^{\prime} \mathrm{E}$ & \\
\hline & Pathum Thani-Lad Lumkeaw & $14^{\circ} \mathrm{O} 2^{\prime} \mathrm{N} 100^{\circ} 24^{\prime} \mathrm{E}$ & \\
\hline \multirow[t]{6}{*}{ Deltamethrin } & Phang-Nga- Keuk-kak & $8^{\circ} 41^{\prime} 47.2^{\prime \prime} \mathrm{N} 98^{\circ} 15^{\prime} 28.6^{\prime \prime} \mathrm{E}$ & Komalamisra et al. 2006 [114] \\
\hline & Nonthaburi-Baan Suan & $13^{\circ} 51^{\prime} \mathrm{N} 100^{\circ} 29^{\prime} \mathrm{E}$ & Sathantriphop et al. 2006 [106] \\
\hline & Nonthaburi-Muenng & $13^{\circ} 50^{\prime} \mathrm{N} 100^{\circ} 29^{\prime \prime} \mathrm{E}$ & Sathantriphop et al. 2006 [113] \\
\hline & Tak-Mae Sot & $16^{\circ} 47^{\prime} \mathrm{N} 98^{\circ} 36^{\prime} \mathrm{E}$ & \\
\hline & Bangkok-Pom Prab Satru Phai & $13^{\circ} 45^{\prime} \mathrm{N} 100^{\circ} 30^{\prime} \mathrm{E}$ & \\
\hline & (laboratory strain) in 1978 & & \\
\hline \multirow[t]{2}{*}{ Etofenprox } & Chiang Mai-Mueang & $13^{\circ} 19^{\prime} \mathrm{N} 100^{\circ} 55^{\prime} \mathrm{E}$ & Somboon et al. 2003 [93] \\
\hline & Nan-Tha Wang Pha & $19^{\circ} 7^{\prime} 35^{\prime \prime} \mathrm{N} 100^{\circ} 48^{\prime} 53^{\prime \prime} \mathrm{E}$ & \\
\hline Fenitrothion & Nonthaburi-Baan Suan & $13^{\circ} 51^{\prime} \mathrm{N} 100^{\circ} 29^{\prime} \mathrm{E}$ & Sathantriphop et al. 2006 [106] \\
\hline Malathion & Nan-Tha Wang Pha & $19^{\circ} 7^{\prime} 35^{\prime \prime} \mathrm{N} 100^{\circ} 48^{\prime} 53^{\prime \prime} \mathrm{E}$ & Somboon et al. 2003 [93] \\
\hline \multirow[t]{4}{*}{ Permethrin } & Phang-Nga Keuk-kak & $8^{\circ} 41^{\prime} 47.2^{\prime \prime} \mathrm{N} 98^{\circ} 15^{\prime} 28.6^{\prime \prime} \mathrm{E}$ & Komalamisra et al. 2006 [114] \\
\hline & Nonthaburi-Baan Suan & $13^{\circ} 51^{\prime} \mathrm{N} 100^{\circ} 29^{\prime} \mathrm{E}$ & Sathantriphop et al. 2006 [106] \\
\hline & Nonthaburi-Mueang & $13^{\circ} 50^{\prime} \mathrm{N} 100^{\circ} 31^{\prime} \mathrm{E}$ & Thanispong et al. 2008 [14] \\
\hline & Pathum Thani-Lad Lumkeaw & $14^{\circ} \mathrm{O} 2^{\prime} \mathrm{N} 100^{\circ} 24^{\prime} \mathrm{E}$ & \\
\hline \multirow[t]{2}{*}{ Propoxur } & Nonthaburi-Baan Suan & $13^{\circ} 51^{\prime} \mathrm{N} 100^{\circ} 29^{\prime} \mathrm{E}$ & Sathantriphop et al. 2006 [106] \\
\hline & Nonthaburi-Mueang & $13^{\circ} 50^{\prime} 30^{\prime \prime} \mathrm{N} 100^{\circ} 29^{\prime} 45^{\prime \prime} \mathrm{E}$ & Sathantriphop et al. 2006 [113] \\
\hline
\end{tabular}

many of the assay variations have been reviewed [12]. The World Health Organization developed the first test box to evaluate the excitation ("irritability") of exposed mosquitoes following physical contact with insecticides [115]. This system was subsequently referred to as an "excito-repellency" test box [116]. The test system was further modified by other investigators interested in behavioral avoidance responses to DDT and some of the early synthetic pyrethroids [12,117-121]. A light-proof test chamber was designed to study the irritant response of Anopheles gambiae, an important malaria vector in Africa, to several chemical compounds [122]. One limitation associated with all of these earlier test systems was the procedural difficulty for introducing and removing test specimens with the assay designs. Other concerns were the ability to evaluate various physiological states found in wild-caught mosquitoes and selecting the ideal range of concentrations for chemical evaluation.
Moreover, at the time no universal analysis or set of statistical methods for interpretation of data had been fully accepted nor had any test system been designed to more clearly discriminate between contact excitation and noncontact (spatial) repellency responses [12]. An improved excito-repellency test device that was able to better differentiate between excitation and spatial repellency was developed and first tested against field populations of Anopheles albimanus in Central America [12,19]. Unfortunately, this fixed prototype was cumbersome to handle and required considerable time for attaching the insecticide-treated test papers inside the chamber. Eventually, a more field deployable test system was designed that was collapsible and easily transportable [123,124]. This system has been extensively used to investigate the behavioral responses of various mosquito species in Thailand and elsewhere in Asia [125-128]. Additionally, a more compact, modular assay system called the 


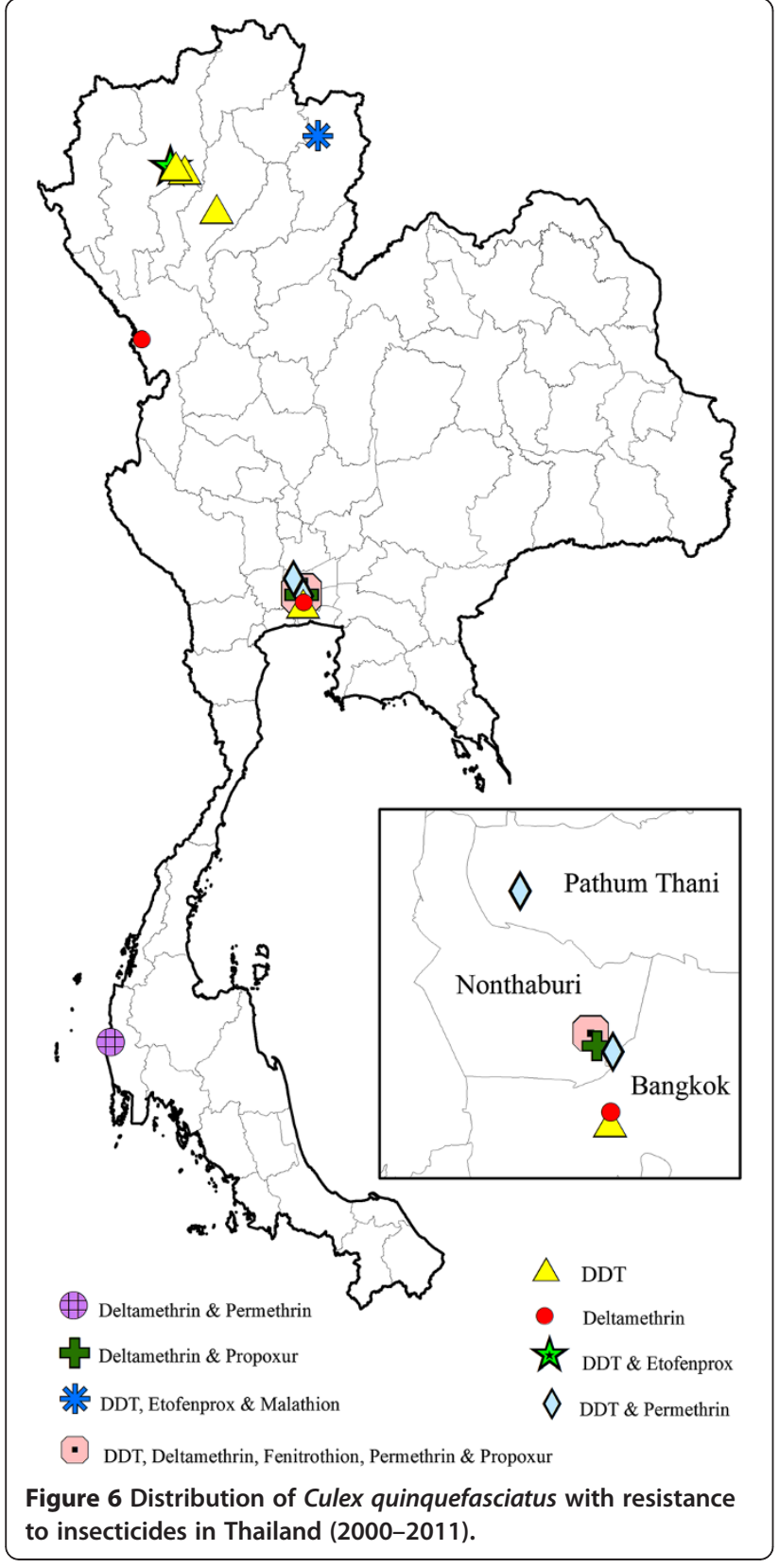

High Through-put Screening System (HITSS) was developed for mass screening of chemicals and adult mosquito responses; including contact irritancy, spatial repellency, and toxicity depending on the specific design set-up [129]. This modular system is of a reduced size compared to previous excito-repellency box devices and greatly minimizes the treated surface area required thereby reducing the amount of chemical required for handling and testing. This system is now approved by WHOPES for testing efficacy of new active ingredients intended for spatial repellent [45].
To better approximate insect behaviors in natural field settings, numerous experiments have been made over many decades using specially constructed experimental huts [4,31,121,130-136]. Most experimental hut studies have been conducted to observe the behavior of Anopheles mosquitoes; however, Grieco et al. [4] successfully demonstrated all 3 chemical actions could be observed in experimental huts using Ae. aegypti as a model system. The results obtained from both laboratory and field studies can help facilitate the choice of the most effective chemicals and measures to control house-frequenting adult mosquitoes. The behavioral responses of Anopheles species to various compounds are provided in Table 6 and those for Aedes aegypti and Culex quinquefasciatus in Table 7.

\section{Behavioral avoidance to insecticides \\ DDT}

Unlike physiological resistance, accurately measuring behavioral responses remains elusive and difficult to detect. Some agricultural and medically important insects, including malaria vectors, have allegedly demonstrated what has been termed "behavioral resistance" following repeated exposure to sub-lethal concentrations of DDT [30]. However, "behavioral avoidance" is preferred terminology as it is an innate, involuntary response to an external stimuli rather than a permanent, geneticallybased shift in behavior as the development of apparent fixed behavioral changes because of insecticide selective pressure has not been sufficiently documented to occur under natural conditions [143]. The early observations on behavioral responses of mosquitoes focused on DDT and have been investigated using experimental huts and excito-repellency box (ERB) test systems. The first study on the irritant effect of DDT residual deposits was conducted using Anopheles quadrimaculatus where females were found to be irritated shortly after making contact with the treated surfaces resulting in a rapid escape response from a treated house before taking a blood meal [144]. Subsequent observations found that An. quadrimaculatus often received a lethal dose from this brief contact and perished within 24 hours [145]. Unfortunately, these studies were conducted without having a matched control (untreated houses) for comparison. Furthermore, the high mortality seen with $A n$. quadrimaculatus may have been caused by further contact with toxic ingredients while attempting to leave a treated house through the small portals of the experimental hut [143]. In studies with Anopheles albimanus in Panama, Trapido [146] concluded that wild-caught mosquitoes lacking re-exposure to DDT for a long period of time, showed the same susceptibility levels to DDT as those from a laboratory colony with no history of previous exposure. Malaria vectors in some countries (e.g., Brazil, Thailand) have apparently never 
developed resistance to DDT $[125,130]$ despite years of extensive use inside houses, suggesting that the particular mosquito populations avoid making direct physical contact with the chemical, thereby precluding any selection for resistance. Table 6 lists Anopheles species and levels of behavioral responses to DDT and synthetic pyrethroids in Thailand.

In Thailand, the irritant and repellent effects of DDT were demonstrated against two populations of Anopheles minimus (sensu lato) using an ERB test system [125]. A study comparing 2 members of the Minimus Complex; An. minimus (species A) and Anopheles harrisoni (species $C$ ) found that DDT produced a rapid and striking irritant response in both species, while repellency was more pronounced in An. minimus [127]. With Ae. aegypti, it has been suggested an association exists between increased levels of physiological resistance to DDT and a greater suppression of both excitation and repellency responses, yet the overall avoidance behavior remains a significant response compared to non-exposed controls [140].

\section{Pyrethroids}

Numerous studies have found that synthetic pyrethroids clearly elicit a range of excitation and repellent effects on many insect species that also typically result in mosquitoes moving away ('avoidance') from insecticide sprayed surfaces $[30,147,148]$. The extensive and continuing use of pyrethroids should serve as an incentive to intensify investigations on the operational significance of pyrethroid-induced avoidance behavior in mosquito vectors and other arthropods. Given the important role of indoor residual spraying of homes as a means of reducing risk of malaria transmission, the consequence of excitation and repellent actions should be well defined for specific malaria vectors in defined locations before and after beginning any large scale control program. Following the refinement of the ERB test system and development of the HITSS assay, both that allow the discrimination of 2 primary types of behavioral actions [12], a series of important findings on excitorepellency behavior in Anopheles mosquitoes have been reported in Thailand $[19,70,125,127,137,138,142]$. In general, synthetic pyrethroids consistently result in significantly stronger contact irritant responses in Anopheles compared to repellency (Table 6). For example, lambda-cyhalothrin and deltamethrin act as strong excitatory agents on test populations of $A n$. minimus while showing relatively weak repellent activity [125]. Pothikasikorn et al. [127] also confirmed that An. minimus and An. harrisoni showed a rapid escape response to contact with lambda-cyhalothrin and deltamethrin, while repellency remained significant but lagged in intensity. Chareonviriyaphap et al. [70] described the excito-repellent actions of deltamethrin in four different Anopheles species, all representing important malaria vectors in Thailand, showing deltamethrin can produce a pronounced irritant response relative to a slower, weaker spatial repellency. Although repellency was less outstanding an escape response compared to irritancy, the repellent action and escape responses were still statistically significant compared to the matched paired controls (minus active ingredient).

Numerous behavioral responses of Ae. aegypti populations exposed to a series of pyrethroids (deltamethrin, permethrin, alpha-cypermethrin, cyphenothrin, d-tetramethrin and tetramethrin) have been investigated in depth in Thailand (Table 7). In general, all tested populations have exhibited moderate to strong irritancy when compared to repellency $[97,101,129,140,142]$. Far fewer numbers of populations of $C x$. quinquefasciatus have been tested against the 3 principal classes of insecticides used in vector control; pyrethroids (deltamethrin), organophosphates (fenitrothion) and carbamates (propoxur), yet again, contact excitation appears stronger than repellency against all three compounds (Table 7). However, marked differences in behavioral responses have been seen between different mosquito populations, active ingredients and concentrations. Prominent irritant responses were observed in a long-established colony population exposed to deltamethrin and fenitrothion as compared to two field populations of the same species [106], demonstrating the importance of testing field populations as opposed to long-colonized material.

The behavioral responses to insecticides by mosquitoes are important components of a chemical's overall effectiveness and value in reducing human-vector contact and disease transmission. To date, there is no convincing evidence of fixed behavioral 'resistance' developing in mosquito species to continuous or intermittent exposure to insecticides [149]. Rather, the majority of data supports that a mosquito's actions are part of an innate involuntary behavioral repertoire involving reflex stimulus-response mechanisms. Behavioral responses can be split into 2 distinct categories, stimulus-dependent and stimulusindependent actions [150]. The term avoidance behavior is generally used to describe actions that are stimulusdependent by some combination of excitation and repellency [131].

A stimulus-dependent action requires sensory stimulation of the insect in order for an avoidance behavior to occur. In general, this form of avoidance enables the insect to detect a chemical after making direct contact but before acquiring a lethal dose [143] or detection of vapor-phase molecules of the active ingredient in the air that initiates repellency (deterrence). On the other hand, a stimulus-independent response does not require direct sensory stimulation of the insect for avoidance to occur but rather involves other natural behavioral components such as exophily (outside resting) or zoophily (non- 
Table 6 Evidence of behavioral avoidance responses to insecticides in Anopheles populations in Thailand (2000-2011)

\begin{tabular}{|c|c|c|c|c|c|}
\hline Species & Field/Lab & Dose & Insecticide & Location & Published source \\
\hline & & & & (province-district) & \\
\hline \multirow[t]{3}{*}{ Anopheles minimus $A^{*}$} & Lab & $2.00 \mathrm{~g} / \mathrm{m}^{2}$ & DDT & Phrae-Rong Klang & Chareonviriyaphap et al. 2001 [125] \\
\hline & & $0.0625 \mathrm{~g} / \mathrm{m}^{2}$ & Deltamethrin & Phrae-Rong Klang & \\
\hline & & $0.0369 \mathrm{~g} / \mathrm{m}^{2}$ & Lambdacyhalothrin & Phrae-Rong Klang & \\
\hline \multirow[t]{3}{*}{ Anopheles minimus complex } & Field & $2.00 \mathrm{~g} / \mathrm{m}^{2}$ & DDT & Kanchanaburi-Pu Teuy & \\
\hline & & $0.0625 \mathrm{~g} / \mathrm{m}^{2}$ & Deltamethrin & Kanchanaburi-Pu Teuy & \\
\hline & & $0.0369 \mathrm{~g} / \mathrm{m}^{2}$ & Lambdacyhalothrin & Kanchanaburi-Pu Teuy & \\
\hline Anopheles minimus $A^{*}$ & Lab & $0.02 \mathrm{~g} / \mathrm{m}^{2}$ & Deltamethrin & Phrae-Rong Klang & Chareonviriyaphap et al. 2004 [70] \\
\hline Anopheles dirus B十 & Lab & $0.02 \mathrm{~g} / \mathrm{m}^{2}$ & Deltamethrin & Chantaburi-Ban Paung & \\
\hline Anopheles minimus complex & Field & $0.02 \mathrm{~g} / \mathrm{m}^{2}$ & Deltamethrin & Kanchanaburi-Pu Teuy & \\
\hline Anopheles maculatus B $\neq$ & Lab & $0.02 \mathrm{~g} / \mathrm{m}^{2}$ & Deltamethrin & Tak-Mae Sot & \\
\hline Anopheles swadwongporni & Field & $0.02 \mathrm{~g} / \mathrm{m}^{2}$ & Deltamethrin & Kanchanaburi-Pu Teuy & \\
\hline Anopheles dirus complex & Field & $0.02 \mathrm{~g} / \mathrm{m}^{2}$ & Deltamethrin & Kanchanaburi-Pu Teuy & \\
\hline \multirow[t]{3}{*}{ Anopheles minimus $\mathrm{A}^{*}$} & Field & $2.00 \mathrm{~g} / \mathrm{m}^{2}$ & DDT & Tak-Mae-Sot & Potikasikorn et al. 2005 [127] \\
\hline & & $0.02 \mathrm{~g} / \mathrm{m}^{2}$ & Deltamethrin & Tak-Mae-Sot & \\
\hline & & $0.03 \mathrm{~g} / \mathrm{m}^{2}$ & Lambdacyhalothrin & Tak-Mae-Sot & \\
\hline \multirow[t]{3}{*}{ Anopheles minimus $C \S$} & Field & $2.00 \mathrm{~g} / \mathrm{m}^{2}$ & DDT & Kanchanaburi-Pu Teuy & \\
\hline & & $0.02 \mathrm{~g} / \mathrm{m}^{2}$ & Deltamethrin & Kanchanaburi-Pu Teuy & \\
\hline & & $0.03 \mathrm{~g} / \mathrm{m}^{2}$ & Lambdacyhalothrin & Kanchanaburi-Pu Teuy & \\
\hline \multirow[t]{2}{*}{ Anopheles maculatus } & Field & $2.00 \mathrm{~g} / \mathrm{m}^{2}$ & DDT & Kanchanaburi-Pu Teuy & Muenworn et al. 2006 [137] \\
\hline & & $0.5 \mathrm{~g} / \mathrm{m}^{2}$ & Permethrin & Kanchanaburi-Pu Teuy & \\
\hline \multirow[t]{2}{*}{ Anopheles swadwongporni } & Field & $2.00 \mathrm{~g} / \mathrm{m}^{2}$ & DDT & Kanchanaburi-Pu Teuy & \\
\hline & & $0.5 \mathrm{~g} / \mathrm{m}^{2}$ & Permethrin & Kanchanaburi-Pu Teuy & \\
\hline \multirow[t]{3}{*}{ Anopheles minimus $\mathrm{A}^{*}$} & Field & $0.04 \mathrm{~g} / \mathrm{m}^{2}$ & Cypermethrin & Kanchanaburi-Pu Teuy & Pothikasikorn et al. 2007 [138] \\
\hline & & $0.04 \mathrm{~g} / \mathrm{m}^{2}$ & Carbaryl & Kanchanaburi-Pu Teuy & \\
\hline & & $0.19 \mathrm{~g} / \mathrm{m}^{2}$ & Malathion & Kanchanaburi-Pu Teuy & \\
\hline \multirow[t]{3}{*}{ Anopheles minimus $C \S$} & Field & $0.04 \mathrm{~g} / \mathrm{m}^{2}$ & Cypermethrin & Kanchanaburi-Pu Teuy & \\
\hline & & $0.04 \mathrm{~g} / \mathrm{m}^{2}$ & Carbaryl & Kanchanaburi-Pu Teuy & \\
\hline & & $0.19 \mathrm{~g} / \mathrm{m}^{2}$ & Malathion & Kanchanaburi-Pu Teuy & \\
\hline Anopheles harrisoni & Field & $0.03 \mathrm{~g} / \mathrm{m}^{2}$ & Bifenthrin & Kanchanaburi-Pu Teuy & Tisgratog et al. 2011 [139] \\
\hline Anopheles minimus & Field & $0.03 \mathrm{~g} / \mathrm{m}^{2}$ & Bifenthrin & Tak-Mae Sot & \\
\hline
\end{tabular}

With the exception of one population (An. minimus s.s. in Tak Province/Mae Sot District to DDT) all species show contact excitation (irritancy) as the predominant response versus noncontact spatial repellency.

*Published as Anopheles minimus A, now formally named Anopheles minimus s.s.; †Anopheles dirus B now as Anopheles crascens; $¥$ Anopheles maculatus B as Anopheles maculatus s.s. [66] and § Anopheles minimus C as Anopheles harrisoni.

human blood preference) in which an insect avoids the exposure to a chemical by preferentially utilizing habitats without insecticides present [151]. This second type of response has also been included as innate, geneticallydriven phenotypic and genotypic behaviors [152]. Stimulus-dependent behavioral responses include the avoidance behaviors discussed in this review.

\section{Conclusions}

A number of synthetic pyrethroids, i.e. allethrin, deltamethrin, permethrin, cypermethrin, alpha-cypermethrin, and cyfluthrin, are commonly used by home owners, private business and in the public sector to control both household pests and medically important insect vectors. In the past, the prevailing practice has been to classify chemicals simply based on their toxicity (killing) profile of insects. We have preferred the use of the term "chemical" in place of "insecticide" as it is more appropriate for recognizing the multiple actions these active ingredients have on mosquitoes via toxicity alone. This also recognizes that chemicals protect humans from the mosquito blood feeding by at least 3 different primary 
Table 7 Evidence of behavioral avoidance responses in Aedes aegypti and Culex quinquefasciatus to insecticides in Thailand $(2000$ - 2011)

\begin{tabular}{|c|c|c|c|c|c|}
\hline Species & Field/Lab & Dose & Insecticide & Location & Published source \\
\hline Aedes aegypti & Field & $0.02 \mathrm{~g} / \mathrm{m}^{2}$ & Deltamethrin & Bangkok & Kongmee et al. 2004 [126] \\
\hline Aedes aegypti & Field & $0.02 \mathrm{~g} / \mathrm{m}^{2}$ & Deltamethrin & Pathum Thani & \\
\hline Aedes aegypti & Field & $0.02 \mathrm{~g} / \mathrm{m}^{2}$ & Deltamethrin & Nonthaburi & \\
\hline Aedes aegypti & Lab & $0.02 \mathrm{~g} / \mathrm{m}^{2}$ & Deltamethrin & Ayutthaya & \\
\hline Aedes aegypti & Lab & $0.02 \mathrm{~g} / \mathrm{m}^{2}$ & Deltamethrin & Bangkok & \\
\hline Aedes aegypti & Lab & $0.25 \mathrm{~g} / \mathrm{m}^{2}$ & Permethrin & Nonthaburi & Paeporn et al. 2007 [97] \\
\hline \multirow[t]{2}{*}{ Aedes aegypti } & Field & $0.025 \mathrm{~g} / \mathrm{m}^{2}$ & Alphacypermethrin & Kanchanaburi & Thanispong et al. 2009 [140] \\
\hline & & $2.00 \mathrm{~g} / \mathrm{m}^{2}$ & DDT & Kanchanaburi & \\
\hline \multirow[t]{2}{*}{ Aedes aegypti } & Field & $0.025 \mathrm{~g} / \mathrm{m}^{2}$ & Alphacypermethrin & Chiang Mai & \\
\hline & & $2.00 \mathrm{~g} / \mathrm{m}^{2}$ & DDT & Chiang Mai & \\
\hline \multirow[t]{2}{*}{ Aedes aegypti } & Lab & $0.025 \mathrm{~g} / \mathrm{m}^{2}$ & Alphacypermethrin & USDA & \\
\hline & & $2.00 \mathrm{~g} / \mathrm{m}^{2}$ & DDT & USDA & \\
\hline \multirow[t]{4}{*}{ Aedes aegypti } & Field & $0.010 \%$ & Deltamethirn & Kanchanaburi & Mongkalagoon et al. 2009 [141] \\
\hline & & $0.0113 \%$ & Cyphenothrin & Kanchanaburi & \\
\hline & & $2.091 \%$ & d-Tetramethrin & Kanchanaburi & \\
\hline & & $2.377 \%$ & Tetramethrin & Kanchanaburi & \\
\hline \multirow[t]{4}{*}{ Aedes aegypti } & Field & $0.05 \%$ & Alphacypermethrin & Chiang Mai & Thanispong et al. 2010 [142] \\
\hline & & $0.05 \%$ & Deltamethrin & Chiang Mai & \\
\hline & & $0.25 \%$ & Permethrin & Chiang Mai & \\
\hline & & $4.00 \%$ & DDT & Chiang Mai & \\
\hline \multirow[t]{4}{*}{ Aedes aegypti } & Field & $0.05 \%$ & Alphacypermethrin & Kanchanaburi & \\
\hline & & $0.05 \%$ & Deltamethrin & Kanchanaburi & \\
\hline & & $0.25 \%$ & Permethrin & Kanchanaburi & \\
\hline & & $0.25 \%$ & DDT & Kanchanaburi & \\
\hline \multirow[t]{4}{*}{ Aedes aegypti } & Field & $0.05 \%$ & Alphacypermethrin & Khon Kean & \\
\hline & & $0.05 \%$ & Deltamethrin & Khon Kean & \\
\hline & & $0.25 \%$ & Permethrin & Khon Kean & \\
\hline & & $4.00 \%$ & DDT & Khon Kean & \\
\hline \multirow[t]{4}{*}{ Aedes aegypti } & Field & $0.05 \%$ & Alphacypermethrin & Nonthaburi & \\
\hline & & $0.05 \%$ & Deltamethrin & Nonthaburi & \\
\hline & & $0.25 \%$ & Permethrin & Nonthaburi & \\
\hline & & $4.00 \%$ & DDT & Nonthaburi & \\
\hline \multirow[t]{4}{*}{ Aedes aegypti } & Field & $0.05 \%$ & Alphacypermethrin & Songkhla & \\
\hline & & $0.05 \%$ & Deltamethrin & Songkhla & \\
\hline & & $0.25 \%$ & Permethrin & Songkhla & \\
\hline & & $4.00 \%$ & DDT & Songkhla & \\
\hline \multirow[t]{4}{*}{ Aedes aegypti } & Field & $0.05 \%$ & Alphacypermethrin & Satun & \\
\hline & & $0.05 \%$ & Deltamethrin & Satun & \\
\hline & & $0.25 \%$ & Permethrin & Satun & \\
\hline & & $4.00 \%$ & DDT & Satun & \\
\hline
\end{tabular}


Table 7 Evidence of behavioral avoidance responses in Aedes aegypti and Culex quinquefasciatus to insecticides in Thailand (2000 - 2011) (Continued)

\begin{tabular}{|c|c|c|c|c|c|}
\hline \multirow[t]{3}{*}{ Culex quinquefasciatus } & \multirow[t]{3}{*}{ Field } & $0.02 \mathrm{~g} / \mathrm{m}^{2}$ & Deltamethrin & Nonthaburi & \multirow{6}{*}{ Sathantriphop et al. 2006 [113] } \\
\hline & & $0.20 \mathrm{~g} / \mathrm{m}^{2}$ & Fenitrothion & Nonthaburi & \\
\hline & & $0.20 \mathrm{~g} / \mathrm{m}^{2}$ & Propoxur & Nonthaburi & \\
\hline \multirow[t]{3}{*}{ Culex quinquefasciatus } & \multirow[t]{3}{*}{ Field } & $0.02 \mathrm{~g} / \mathrm{m}^{2}$ & Deltamethrin & Tak & \\
\hline & & $0.20 \mathrm{~g} / \mathrm{m}^{2}$ & Fenitrothion & Tak & \\
\hline & & $0.20 \mathrm{~g} / \mathrm{m}^{2}$ & Propoxur & Tak & \\
\hline \multirow[t]{3}{*}{ Culex quinquefasciatus } & \multirow[t]{3}{*}{ Lab } & $0.02 \mathrm{~g} / \mathrm{m}^{2}$ & Deltamethrin & Nonthaburi & \\
\hline & & $0.20 \mathrm{~g} / \mathrm{m}^{2}$ & Fenitrothion & Nonthaburi & \\
\hline & & $0.20 \mathrm{~g} / \mathrm{m}^{2}$ & Propoxur & Nonthaburi & \\
\hline
\end{tabular}

All species show contact excitation (irritancy) as the predominant response versus noncontact spatial repellency.

actions: excitation ('irritancy'), repellency and toxicity [4,32]. Historically, the vast majority of studies have focused on the direct toxicological (mortality) responses (susceptibility and resistance patterns) of chemicals on mosquito populations; whereas very little emphasis was placed on the vector's behavior in response to sub-lethal exposures to various compounds. Knowledge of a mosquito's normal and chemical-induced behavioral responses under varying conditions (e.g., age, physiological state, parity, etc.) is important in the prioritization and design of appropriate vector prevention and control strategies. The development of insecticide resistance in insect pests and disease vectors worldwide is increasing on an alarming scale. However, patterns of resistance is not uniform across all areas, with some vector populations having had low instances of resistance in spite of long-term use of chemicals used to control them, suggesting that behavioral responses may likely be playing a significant role influencing how certain chemicals perform to interrupt human-vector contact while reducing the selection pressure on target insects for developing physiological resistance [32]. In Thailand, populations of Ae. aegypti showing patterns of low-grade resistance to pyrethroids may indicate that resistance is increasing in a particular population; it is also possible that resistance may be declining subsequent to diminution of selective pressure. We postulate that behavioral avoidance may result in a third alternative - a low level equilibrium of physiological resistance.

Avoidance responses in which normal host-seeking and biting activities are disrupted pose a challenge to wild adult mosquitoes on a tight energy budget to fly longer and greater distances than would have otherwise occurred. This might have an impact on its survival or fecundity thereby impacting both subsequent vector population densities and disease transmission. Behavioral avoidance of insecticides may have similar effects, should insecticide coverage be sufficiently high. Recent evidence of apparent decreasing densities of vectors and reduction of malaria in Africa poses questions as to the reasons or mechanisms behind the population reductions [153]. The debate whether repellency or toxicity is the more favored attribute of an insecticide [32,33,154], may likely depend on the specific vector species being targeted for control. As IRS and ITN chemical treatments gradually reach sublethal doses what are the implications on the biology of mosquito vectors, their populations and the diseases they transmit? Is behavioral avoidance a liability or an asset in the control of new infections? For example, ITNs have been implicated in the massive reduction in An. darlingi in Suriname [155], whether due to direct killing effects or deterrence. On the other hand, deterrence may impact transmission dynamics that possibly exacerbate control efforts to suppress transmission [156,157]. One example of attempting to expolit contact irritant responses of Ae. aegypti has been the evaulation of sublethal concentrations and focal application of pyrethroid chemicals for the distruption of normal blood feeding and resting behavior [25].

Whether a chemical is acting by direct contact with a mosquito or spatially from a distance, both behavioral responses are stimulus-response actions that result in distinct movement away from an area where the chemical is present. We now have various types of excitorepellency test systems (e.g., ERB and HITSS assays) that can accurately measure the behavioral responses of mosquitoes to chemicals. WHOPES has very recently recommended a battery of test procedures to better address the efficacy and mode of action of active ingredients intended for "spatial repellent" applications [158]. However, the "proof of principle" that spatial repellency can effectively reduce malaria or dengue transmission, both indoors and out, merits further investigation.

To conclude, we believe that a better understanding and evaluation of physiological and behavioral responses by mosquitoes to chemicals are an important, if not critical, components of any disease control operation dependent on 
this abatement strategy. We recommend that in addition to establishing a country-wide network of routine resistance monitoring and behavioral response evaluations where vector control is taking place, a greater emphasis is needed to investigate the biochemical and genetic mechanisms responsible for resistance in vector populations. There should also be an expansion of the number of different vector species examined. For example, there have been virtually no investigations on the response to insecticides by the primary culicine vectors of Japanese encephalitis virus or Mansonia species responsible for lymphatic filariasis. Lastly, because of the recent governmental re-organization in Thailand allowing each municipality decision-making authority for how local vector control operations are conducted, including the purchasing insecticides, there is concern that both ineffectual and inefficient vector control practices might result. Such an unstructured and independently managed system might also contribute to an escalation of insecticide resistance. It would be advisable, that clear and enforceable policies that are evidence-based for insecticide selection be directed from the MOPH, Department of Disease Control.

Knowledge of vector/pest susceptibility to pesticides, changing patterns of resistance and their operational implications, will continue to be basic requirements to help guide chemical use in evidence-based, vector-borne disease and pest control programs. Although not an exhaustive compilation, this review has attempted to summarize the current knowledge of how chemicals affect various vector species and populations in Thailand. With this background, it is hoped that operational and research endeavors will benefit from this overview and focus on areas where critical information is lacking.

\section{Abbreviations}

IRS: Indoor residual spraying; ITN: Insecticide-treated netting (bednets); DEET: N, N-diethyl-meta-toluamide; PCO: Pest control operators; MFO: Mixed function oxidases (P-450 mediated monooxygenases); GST: Glutathione Stransferases; ERB: Excito-repellency box; HITSS: High throughput screening system; WHOPES: World Health Organization Pesticide Evaluation Scheme; MOPH: Ministry of Public Health (Thailand).

\section{Competing interests}

The authors declare that they have no competing interests.

\section{Authors' contributions}

TC reviewed the literature and formulated the review. TC wrote the paper with assistance from MJB, WS, MK, VC, and RNK. All authors read and approved the final manuscript.

\section{Acknowledgements}

We thank the Thailand Research Fund (TRF) (RTA5580002) and the Thailand International Development and Cooperation Agency (TICA through the STOP-VEC programme) for providing financial support for this publication. The funders had no role in the data collection and analysis, decision to publish, or preparation of the manuscript.

\section{Author details}

${ }^{1}$ Department of Entomology, Faculty of Agriculture, Kasetsart University, Bangkok 10900, Thailand. 'Public Health \& Malaria Control Department, International SOS, Kuala Kencana, Papua 99920, Indonesia. ${ }^{3}$ Department of
Disease Control, Ministry of Public Health, Chiang Mai 52000, Thailand. ${ }^{4}$ Department of Entomology, Faculty of Agriculture at Kamphaeng Saen Kasetsart University, Kamphaeng Saen Campus, Nakhon Pathom 73140, Thailand. Institut de Recherche pour le Développement (IRD), Maladies Infectieuses et Vecteurs, Ecologie, Génétique, Evolution et Contrôle (MIVEGEC, IRD 224-CNRS 5290 UM1-UM2), Montpellier, France.

Received: 8 August 2013 Accepted: 20 September 2013

Published: 25 September 2013

\section{References}

1. Roberts DR, Andre RG: Insecticide resistance issues in vector-borne disease control. Am J Trop Med Hyg 1994, 50:21-34.

2. Reiter P, Gubler DJ: Surveillance and control of urban dengue vectors. In Dengue and dengue hemorragic fever. Edited by Gubler D, Kuno G. New York: CAB International; 1997:45-60.

3. WHO: Prevention and control and denque and denque haemorrhagic fever: Comprehensive guidelines. New Delhi: World Health Organization Regional Publication; 1999.

4. Grieco JP, Achee NL, Chareonviriyaphap T, Suwonkerd W, Chauhan K, Sardelis MR, Robert DR: A new classification system for the actions of IRS chemicals traditionally used for malaria control. PLoS One 2007, 2:e716. doi:10.1371/journal.pone.0000716.

5. Stein G: Malaria eradication in Thailand. Bangkok, Thailand: USOM; 1970.

6. Prasittisuk C: Present status of malaria in Thailand. Southeast Asian J Trop Med Public Health 1985, 16:141-145.

7. Chareonviriyaphap T, Bangs MJ, Ratanatham S: Status of malaria in Thailand. Southeast Asian J Trop Med Public Health 2000, 31:225-237.

8. Chareonviriyahpap T, Aum-Aung B, Ratanatham S: Current insecticide resistance patterns in mosquito vectors in Thailand. Southeast Asian $J$ Trop Med Public Health 1999, 30:184-194.

9. Bureau of Vector Borne Disease: Ministry of Public Health: Annual Vector Borne Disease Reports. Bangkok, Thailand: Bureau of Vector Borne Disease, Department of Disease Control; 2000.

10. Zaim M, Aitio A, Nakashima N: Safety of pyrethroid-treated mosquito nets. Med Vet Entomol 2000, 14:1-5.

11. WHO: Discrimination concentrations of insecticide for adult mosquitoes. Geneva, Switzerland: World Health Organization; 2009.

12. Roberts DR, Chareonviriyaphap T, Harlan HH, Hshieh P: Methods of testing and analyzing excito-repellency responses of malaria vectors to insecticides. J Am Mosa Contr Assoc 1997, 13:13-17.

13. Jirakanjanakit N, Rongnoparut P, Saengtharatip S, Chareonviriyaphap T Duchon S, Bellec C, Yoksan S: Insecticide susceptible/resistance status in Aedes (Stegomyia) aegypti and Aedes (Stegomyia) albopictus (Diptera: Culicidae) in Thailand during 2003-2005. J Econ Entomol 2007. 100:545-550.

14. Thanispong K, Sathantriphop S, Chareonviriyaphap T: Insecticide resistance of Aedes aegypti and Culex quinquefasciatus in Thailand. J Pestic Sci 2008, 33:351-356.

15. Ranson H, Burhani J, Lumjuan N, Black WC IV: Insecticide resistance in dengue vectors. 2010. TroplKA.net 1, 0-0.

16. Walker TW, Robert LL, Copeland RA, Githeko AK, Wirtz RA, Githure Jl, Klein TA: Field evaluation of arthropod repellents, deet and a piperidine compound, Al3-37220, against Anopheles funestus and Anopheles arabiensis in western Kenya. J Am Mosa Control Assoc 1996, 12:172-176.

17. Fradin MS, Day JF: Comparative efficacy of insect repellents against mosquito bites. N Engl J Med 2002, 347:13-18.

18. Hemingway J, Hawkes NJ, McCarroll L, Ranson H: The molecular basis of insecticide resistance in mosquitoes. Insect Biochem Mol Biol 2004, 34:653-665

19. Chareonviriyaphap T, Roberts DR, Andre RG, Harlan HJ, Manguin S, Bangs MJ: Pesticide avoidance behavior in Anopheles albimanus, a malaria vector in the Americas. J Am Mosq Control Assoc 1997, 13:171-183.

20. Ranson H, N'Guessan R, Lines J, Moiroux N, Nkuni Z, Corbel V: Pyrethroid resistance in African anopheline mosquitoes: what are the implications for malaria control? Trends Parasitol 2011, 27:91-98.

21. Ditzen M, Pellegrino M, Vosshall LB: Insect odorant receptors are molecular targets of the insect repellent DEET. Science 2008 , 319:1838-1841.

22. WHO: Vector resistance to pesticides. Geneva: World Health Organization; 1992:62. 
23. Roberts DR, Tren R: DDT in malaria control: Roberts and Tren respond. Environ Health Perspect 2010, 118:A283.

24. Roberts DR: Insecticide Repellency in Malaria Vector Control: a Position Paper. Arlington, VA: United States Agency for International Development; 1993:72. VBC. Report No. 81131 VBC. Report No. 81131.

25. Manda H, Shah P, Polsomboon S, Chareonviriyaphap T, Castro-Llanos F, Morrison A, Burrus RG, Grieco JP, Achee NL: Contact irritant responses of Aedes aegypti using sublethal concentration and focal application of pyrethroid chemicals. PLOS NTD 2013, 7:e2074.

26. Kennedy JS: The excitant and repellent effects on mosquitoes of sublethal contacts with DDT. Bull Entomol Res 1947, 37:593-607.

27. Muirhead-Thomson RC: Mosquito Behaviour in relation to Malaria Transmission and Control in the Tropics. London: Arnold; 1951:219.

28. Davidson G: Experiments on the effect of residual insecticides in houses against Anopheles gambiae and Anopheles funestus. Bull Entomol Res 1953, 44:231-254.

29. Dethier VG, Browne LB, Smith CW: The designation of chemicals in terms of the responses they elicit from insects. J Econ Entomol 1960, 53:134-136.

30. Lockwood JA, Sparks TC, Story RN: Evolution of insect resistance to insecticides: a reevaluation of the roles of physiology and behavior Bull Entomol Soc Am 1984, 30:41-51.

31. Grieco JP, Achee NL, Andre RG, Roberts DR: A comparison study of house entering and exiting behavior of Anopheles vestitipennis (Diptera: Culicidae) using experimental huts sprayed with DDT or deltamethrin in the southern district of Toledo, Belize. C. A. J Vector Ecol 2000, 25:62-73.

32. Roberts DR, Alecrim WD, Hshieh P, Grieco JP, Bangs M, Andre RG, Chareonviriyaphap T: A probability model of vector behavior: Effects of DDT repellency, irritancy, and toxicity in malaria control. J Vector Ecol 2000, 25:48-61.

33. Achee NL, Sardelis MR, Dusfour I, Chauhan KR, Grieco JP: Characterization of spatial repellent, contact irritant, and toxicant chemical actions of standard vector control compounds. J Am Mosq Control Assoc 2009, 25:156-167.

34. Paeporn P, Supaphathom K, Srisawat R, Komalamisra N, Deesin V, Yaumphan $\mathrm{P}$, Leeming Sawat $\mathrm{S}$ : Biochemical detection of pyrethroid resistance mechanism in Aedes aegypti in Ratchaburi province, Thailand. Trop Biomed 2004, 21:145-151.

35. Chuaycharoensuk T, Juntarajumnong W, Boonyuan W, Bangs MJ, Akratanakul P, Thammapalo S, Jirakanjanakit N, Tanasinchayakul S, Chareonviriyaphap T: Frequency of pyrethroid resistance in Aedes aegypti and Aedes albopictus (Diptera: Culicidae) in Thailand. J Vector Ecol 2011, 36:204-212

36. Bureau of Vector Borne Disease, Ministry of Public Health: Annual Vector Borne Disease Reports: Bureau of Vector Borne Disease. Bangkok, Thailand: Department of Disease Control; 2008.

37. McGready R, Boel M, Rijken MJ, Ashley EA, Cho T, Moo O, Paw MK, Pimanpanarak M, Hkirijareon L, Carrara VI, Lwin KM, Phyo AP, Turner C, Chu $C S$, van Vugt M, Price RN, Luxemburger C, ter Kuile FO, Tan SO, Proux S, Singhasivanon $\mathrm{P}$, White NJ, Nosten FH: Effect of early detection and treatment on malaria related maternal mortality on the north-western border of thailand 1986-2010. PLOS ONE 2012, 7:b e40244. doi:10.1371/ journal.pone.0040244.

38. Manguin S, Bangs MJ, Pothikasikorn J, Chareonviriyaphap T: Review on global co-transmission of human Plasmodium species and Wuchereria bancrofti by Anopheles mosquitoes. Infect Genet Evol 2010, 10:159-177.

39. Konchom S, Singhasivanon P, Kaewkungwal J, Chupraphawan S, Thimasarn K, Kidson C, Rojanawatsirivet C, Yimsamran S, Looareesuwan S: Trend of malaria incidence in highly endemic provinces along the Thai borders, 1991-2001. Southeast Asian J Trop Med Public Health 2003, 34:486-494.

40. Zhou G, Sirichaisinthop J, Sattabongkot J, Jones J, Bjornstad ON, Yan G, Cui $L$ : Spatio-temporal distribution of Plasmodium falciparum and P. vivax malaria in Thailand. Am J Trop Med Hyg 2005, 72:256-262.

41. Childs DZ, Cattadori IM, Suwonkerd W, Prajakwong S, Boots M: Spatiotemporal patterns of malaria incidence in northern Thailand. Trans R Soc Trop Med Hyg 2006, 100:623-631.

42. WHO: Generic risk assessment model for indoor residual spraying of insecticides. 1st revision. Geneva, Switzerland: World Health Organization; 2011.

43. Sattabongkot J, Tsuboi T, Zollner GE, Sirichaisinthop J, Cui L: Plasmodium vivax transmission: Chances for control? Trends Parasitol 2004, 20:192-198.
44. Gubler DJ: Dengue and dengue hemorrhagic fever. Clin Microbiol Rev 1998, 11:480-496.

45. WHO: Global strategy for dengue prevention and control 2012-2020. Geneva, Switzerland: World Health Organization; 2012.

46. Bureau of Vector Borne Disease, Ministry of Public Health: Annual Vector Borne Disease Reports. Bangkok, Thailand: Bureau of Vector Borne Disease, Department of Disease Control; 2012.

47. Powers AM, Brault AC, Tesh RB, Weaver SC: Re-emergence of chikungunya and o'nyong-nyong viruses: evidence for distinct geographical lineages and distant evolutionary relationships. J Gen Virol 2000, 81:471-479.

48. Pialoux $G$, Gaüzère BA, Jauréguiberry $S$, Strobel M: Chikungunya, an epidemic arbovirosis. Lancet Infect Dis 2007, 7:319-327.

49. Chusri S, Siripaitoon P, Hirunpat S, Silpapojakul K: Short report: Case reports of neuro-chikungunya in southern Thailand. Am J Trop Med Hyg 2011, 85:386-389.

50. Ghosh D, Basu A: Japanese encephalitis - A pathological and clinical perspective. PLOS NTD 2009, 3:e437.

51. Grossman RA, Gould DJ, Smith TJ, Johnsen DO, Pantuwatana S: Study of Japanese encephalitis virus in Chiangmai Valley, Thailand. I. Introduction and study design. Am J Epidemiol 1973, 98:111-120.

52. Solomon T, Dung NM, Kneen R, Gainsborough M, Vaughn DW, Khanh VT: Japanese encephalitis. J Neurol Neurosurg Psychiatry 2000, 68:405-415.

53. Pyke AT, Williams DT, Nisbet DJ, Van den Hurk AF, Taylor CT, Johansen CA, Macdonald J, Hall RA, Simmons RJ, Lee JM, Ritchie SA, Smith GA, Mackenzie JS: The appearance of a second genotype of Japanese encephalitis virus in the Australasian region. Am J Trop Med Hyg 2001, 65:747-753.

54. Tsai TF, Vaughn DW, Solomon T: Flaviviruses (Yellow fever, Dengue, Dengue hemorrhagic fever, Japanese encephalitis, West Nile encephalitis, St. Louis encephalitis, tick-borne encephalitis). In Principles and Practice of Infectious Diseases. 6th edition. Edited by Mandell GL, Bennett JE, Dolin R. Philadelphia: Elsevier Churchill, Livingston; 2005:1926-1950.

55. Nitatpattana N, Dubot-Pérès A, Ar Gouilh M, Souris M, Barbazan P, Yoksan S, de Lamballerie X, Gonzalez JP: Change in Japanese encephalitis virus distribution, Thailand. Emerg Infect Dis 2008, 14:1762-1765

56. Michael E, Bundy DAP: Global mapping of lymphatic filariasis. Parasitol Today 1997, 13:472-476.

57. WHO: Preparing and implementing a national plan to eliminate lymphatic filariasis (in countries where onchocerciasis is not co-endemic). Geneva, Switzerland: World Health Organization; 2000:67.

58. Pothikasikorn J, Bangs MJ, Boonplueang R, Chareonviriyaphap T: Susceptibility of various mosquitoes of Thailand to nocturnal subperiodic Wuchereria bancrofti. J Vector Ecol 2008, 33:313-320.

59. Harinasuta C, Sucharit S, Deesin T, Surathin K, Vutikes S: Bancroftian filariasis in Thailand, a new endemic area. Southeast Asian J Trop Med Public Health 1970, 1:233-245

60. Gould DJ, Bailey CL, Vongpradist S: Implication of forest mosquitoes in the transmission of Wuchereria bancrofti in Thailand. Mosa News 1982, 42:560-564.

61. Green CA, Rattanarithikul R, Pongparit S, Sawadwongporn P, Baimai V: A newly-recognized vector of human malarial parasites in the Oriental region, Anopheles (Cellia) pseudowillmori (Theobald, 1910). Trans $R$ Soc Trop Med Hyg 1991, 85:35-36.

62. Rosenberg $R$, Andre RG, Somchit L: Highly efficient dry season transmission of malaria in Thailand. Trans R Soc Trop Med Hyg 1990, 84:22-28.

63. Rattanarithikul R, Linthicum KJ, Konishi E: Seasonal abundance and parity rates of Anopheles species in southern Thailand. J Am Mosa Control Assoc 1996, 12:75-83.

64. Gould DJ, Esah S, Pranith U: Relation of Anopheles aconitus to malaria transmission in the central plain of Thailand. Trans R Soc Trop Med Hyg 1967, 61:441-442.

65. Maheswary NP, Habib MA, Elias M: Incrimination of Anopheles aconitus Donitz as a vector of epidemic malaria in Bangladesh. Southeast Asian J Trop Med Public Health 1992, 23:798-801.

66. Rattanarithikul R, Harrison BA, Harbach RE, Panthusiri P, Coleman RE: Illustrated keys to the mosquitoes of Thailand IV. Anopheles. Southeast Asian J Trop Med Public Health 2006, 37:1-128.

67. Gubler DJ: Dengue and dengue hemorrhagic fever; its history and resurgence as a global public health problem. In Dengue and Dengue Hemorrhagic Fever. Edited by Gubler DJ, Kuno G. London: CAB International; 1997:1-22. 
68. Christophers SR: Aedes aegypti (L.), the yellow fever mosquito: its life history, bionomics, and structure. London: Cambridge University Press, Cambridge; 1960:739.

69. Smith CE: The history of dengue in tropical Asia and its probable relationship to the mosquito Aedes aegypti. J Trop Med Hyg 1956, 59:243-251.

70. Chareonviriyaphap T, Prabaripai A, Bangs MJ: Excito-repellency of deltamethrin on the malaria vectors, Anopheles minimus, Anopheles dirus, Anopheles swadiwongporni, and Anopheles maculatus, in Thailand. J Am Mosq Control Assoc 2004, 20:45-54

71. Black WC, Hawley WA, Rai KS, Jr Craig GB: Breeding structure of a colonizing species: Aedes albopictus (Skuse) in peninsular Malaysia and Borneo. Heredity 1988, 61:439.

72. Thavara U, Tawatsin A, Chansang C, Kong-ngamsuk W, Paosriwong S, BoonLong J, Rongsriyam Y, Komalamisra N: Larval occurrence, oviposition behavior and biting activity of potential mosquito vectors of dengue on Samui Island, Thailand. J Vector Ecol 2001, 26:172-180.

73. Koehler PG, Castner JL: Bloodsucking Insects. Gainesville: University of Florida; 1997. Cooperative Extension Service, Institute of Food and Agriculture Sciences, EDIS. http://edis.ifas.ufl.edu/IN019 (21 January 2004).

74. Hawley WA: The biology of Aedes albopictus. J Am Mosa Control Assoc 1988, 1:1-39.

75. Turell MJ, Beaman JR, Tammariello RF: Susceptibility of selected strains of Aedes aegypti and Aedes albopictus (Diptera: Culicidae) to chikungunya virus. J Med Entomol 1992, 29:49-53.

76. Schuffenecker I, Iteman I, Michault A, Murri S, Frangeul L, Vaney MC, Lavenir R, Pardigon N, Reynes JM, Pettinelli F, Biscornet L, Diancourt L, Michel S, Duquerroy S, Guigon G, Frenkiel MP, Bréhin AC, Cubito N, Desprès P, Kunst F, Rey F, Zeller H, Brisse $S$ : Genome microevolution of chikungunya viruses causing the Indian Ocean outbreak. PLoS Med 2006, 3:1058-1070.

77. Mitchell CJ, Niebylski ML, Smith GC, Karabatsos N, Martin D, Mutebi JP, Craig GB, Mahler MJ: Isolation and eastern equine encephalitis virus from Aedes albopictus in Florida. Science 1992, 257:526-527.

78. lyengar MO: Filariasis in Thailand. Bull World Health Organ 1953, 9:731-766.

79. Triteeraprapab S, Songtrus J: High prevalence of bancroftian filariasis in Myanmar-migrant workers: a study in Mae Sot District, Tak Province, Thailand. J Med Assoc Thai 1999, 82:733-739.

80. Wiwanitkit V: High prevalence of Filariasis in Myanmar-migrant workers from screening program of a local hospital in a rural district of southern Thailand. Bangkok, Thailand: Chulalongkorn University; 2001. August 8-10, 2001.

81. Gould DJ, Edelman R, Grossman RA, Nisalak A, Sullivan MF: Study of Japanese encephalitis virus in Chiangmai Valley, Thailand. IV. Vector studies. Am J Epidemiol 1974, 100:49-56.

82. Burke DS, Leake CJ: Japanese encephalitis. In The Arboviruses: Epidemiology and Ecology. Edited by Monath TP. Boca Raton: CRC Press; 1988.

83. Nitatpattana N, Apiwathnasorn C, Barbazan P, Leemingsawat S, Yoksan S, Gonzalez JP: First isolation of Japanese encephalitis from Culex quinquefasciatus in Thailand. Southeast Asian J Trop Med Public Health 2005, 36:875-878

84. Solomon T: Flavivirus encephalitis. N Engl J Med 2004, 351:370-378.

85. Jupp PG, Mclntosh BM, dos Santos I, de Moor P: Laboratory vector studies on six mosquito and one tick species with chikungunya virus. Trans R Soc Trop Med Hyg 1981, 75:15-19.

86. Jupp PG, Mclntosh BM: Aedes furcifer and other mosquitoes as vectors of chikungunya virus at Mica, northeastern Transvaal, South Africa. J Am Mosa Control Assoc 1990, 6:415-420.

87. Lam SK, Chua KB, Hooi PS, Rahimah MA, Kumari S, Tharmaratnam M, Chuah SK, Smith DW, Sampson IA: Chikungunya infection- an emerging disease in Malaysia. Southeast Asian J Trop Med Public Health 2001, 32:447-451.

88. Gratz NG: Critical review of the vector status of Aedes albopictus. Med Vet Entomol 2004, 18:215-227.

89. Reiskind MH, Pesko K, Westbrook CJ, Mores CN: Susceptibility of Florida mosquitoes to infection with Chikungunya virus. Am J Trop Med Hyg 2008, 78:422-425.

90. Vazeille M, Moutailler S, Coudrier D, Rousseaux C, Khun H, Huerre M, Thiria J, Dehecq JS, Fontenille D, Schuffenecker I, Despres P, Failloux $A B$ : Two Chikungunya isolates from the outbreak of La Reunion (Indian Ocean) exhibit different patterns of infection in the mosquito. Aedes albopictus. PLoS One 2007, 2:e1168.
91. Vazeille M, Moutailler S, Pages F, Jarjaval F, Failloux AB: Introduction of Aedes albopictus in Gabon: What consequences for dengue and chikungunya transmission? Trop Med Int Health 2008, 13:1176-1179.

92. Higgs S: The 2005-2006 Chikungunya epidemic in the Indian Ocean. Vector Borne Zoonotic Dis 2006, 6:115-116.

93. Somboon P, Prapanthadara LA, Suwonkerd W: Insecticide susceptibility tests of Anopheles minimus s.l., Aedes aegypti, Aedes albopictus, and Culex quinquefasciatus in northern Thailand. Southeast Asian J Trop Med Public Health 2003, 34:87-93.

94. Paeporn P, Suphapathom K, Boonyabancha S, Phan-Urai P: Efficacy of aerosol insecticide product. Bull Dep Med Sci 1996, 38:37-43.

95. Hemingway J, Ranson $\mathrm{H}$ : Insecticide resistance in insect vectors of human disease. Annu Rev Entomol 2000, 45:371-391.

96. Prapanthadara L, Koottathep S, Promtet N, Suwonkerd W, Ketterman AJ, Somboon P: Correlation of glutathione S-transferase and DDT dehydrochlorinase activities with DDT susceptibility in Anopheles and Culex mosquitos from northern Thailand. Southeast Asian J Trop Med Public Health 2000, 31:111-118.

97. Paeporn P, Supaphathorm K, Sathantriphop S, Chareonviriyaphap T, Yaicharoen R: Behavioural responses of deltamethrin- and permethrinresistant strains of Aedes aegypti when exposed to permethrin in an excito-repellency test system. Dengue Bulletin 2007, 31:153-159.

98. Ponlawat A, Scott JG, Harrington LC: Insecticide susceptibility of Aedes aegypti and Aedes albopictus across Thailand. J Med Entomol 2005, 42:821-825.

99. Paeporn P, Supaphathom K, Sathantriphop S: Insecticide susceptibility of Aedes aegypti in different parts of Thailand, 2006-2010. J Vector Borne Dis 2010, 17:8-16.

100. Satimai $W$ : The study of using and insecticide resistant in the eastern Thailand. J Vector Borne Dis 2010, 7:18-30

101. Chareonviriyaphap T, Kongmee M, Bangs MJ, Sathantriphop S, Meunworn V, Akratanakul P: Influence of nutritional and physiological status on behavioral responses of Aedes aegypti (Diptera: Culicidae) to deltamethrin and cypermethrin. J Vector Ecol 2006, 31:89-101.

102. Prapanthadara L, Promtet N, Koottathep S, Somboon P, Suwonkerd W, McCarroll L, Hemingway J: Mechanisms of DDT and permethrin resistance in Aedes aegypti from Chiang Mai, Thailand. Dengue Bulletin 2002, 26:185-189.

103. Lumjuan N, McCarroll L, Prapanthadara LA, Hemingway J, Ranson H: Elevated activity of an Epsilon class glutathione transferase confers DDT resistance in the dengue vector, Aedes aegypti White star. Insect Biochem Mol Biol 2005, 35:861-871.

104. Prapanthadara L, Reunkum W, Leelapat P, Suwan W, Yanola J, Somboon $P$ : Glutathione S-transferase Isoenzymes and the DDTase Activity in Two DDT-resistant Strains of Aedes aegypti. Dengue Bulletin 2005, 29:183-191

105. Yaicharoen R, Kiatfuengfoo R, Chareonviriyaphap T, Rongnoparut $P$ : Characterization of deltamethrin resistance in field populations of Aedes aegypti in Thailand. J Vector Ecol 2005, 30:144-150.

106. Sathantriphop S, Paeporn P, Supaphathom K: Detection of insecticides resistance status in Culex quinquefasciatus and Aedes aegypti to four major groups of insecticides. Trop Biomed 2006, 23:97-101.

107. Rajatileka S, Black WC, Saavedra-Rodriguez K, Trongtokit Y, Apiwathnasorn C, McCall PJ, Ranson H: Development and application of a simple colorimetric assay reveals widespread distribution of sodium channel mutations in Thai populations of Aedes aegypti. Acta Trop 2008, 108:54-57.

108. Pethuan S, Jirakanjanakit N, Saengtharatip S, Chareonviriyaphap T, Kaewpa $D$, Rongnoparut P: Biochemical studies of insecticide resistance in Aedes (Stegomyia) aegypti and Aedes (Stegomyia) albopictus (Diptera: Culicidae) in Thailand. Trop Biomed 2007, 24:7-15.

109. Narksuwan M, Aumauang B, Poolthin S: Susceptibility to Deltamethrin of Aedes aegypti L. from 13 provinces in Thailand. J Vector Borne Dis 2008 5:45-56.

110. Saelim V, Brogdon WG, Rojanapremsuk J, Suvannadabba S, Pandii W, Jones JW, Sithiprasasna R: Bottle and biochemical assays on temephos resistance in Aedes aegypti in Thailand. Southeast Asian J Trop Med Public Health 2005, 36:417-425.

111. Jirakanjanakit N, Saengtharatip S, Rongnoparut P, Duchon S, Bellec C, Yoksan S: Trend of temephos resistance in Aedes (Stegomyia) mosquitoes inThailand during 2003-2005. Environ. Entomol 2007, 36:506-511. 
112. Chadwick PR, Invest JF, Bowron MJ: An example of cross resistance to pyrethroids in DDT resistant Aedes aegypti. Pestic Sci 1977, 8:618-624.

113. Sathantriphop S, Ketavan C, Prabaripai A, Visetson S, Bangs MJ, Akratanakul P, Chareonviriyaphap T: Susceptibility and avoidance behavior by Culex quinquefasciatus Say to three classes of residual insecticides. J Vector Ecol 2006, 31:266-274.

114. Komalamisra N, Trongtokit Y, Palakul K, Prummongkol S, Samung Y, Apiwathnasorn C, Phanpoowong T, Asavanich A, Leemingsawat S: Insecticide susceptibility of mosquitoes invading tsunami-affected areas of Thailand. Southeast Asian J Trop Med Public Health 2006, 37(Suppl 3):118-122

115. WHO: Instruction for determining the susceptibility or resistance of adult mosquitoes to organochlorine insecticides. WHO Tech Rep Ser 1970, 443:47-56.

116. Rachou RG, Lima MM, Duret JP, Kerr JA: Experiences with the ExcitoRepellency Test Box-Model OPS. New Jersey; 1963:442-447.

117. Bondareva NL, Artem'ev MM, Gracheva GV: Susceptibility and irritability caused by insecticides to malaria mosquitoes in the USSR. Part 1. Anopheles pulcherrimus. Meditsinskaia Parazitologha I Parazitarnye Bolezni (Moskva) 1986, 6:52-55.

118. Charlwood JD, Paraluppi ND: The use of excito-repellency box with Anopheles darlingi Root, An. nuneztovari Gabaldon and Culex pipiens quinquefaciatus Say, obtained from the areas near Manaus, Amazonas. Acta Amazon 1978, 8:605-611.

119. Quinones ML, Suarez MF: Irritability to DDT of natural populations of the primary malaria vectors in Colombia. J Am Mosq Control Assoc 1989, 5:56-59.

120. Ree HI, Loong KP: Irritability of Anopheles farauti, Anopheles maculatus, and Culex quinquefasciatus to permethrin. Jap J Sanit Zool 1989, 40:47-51.

121. Rozendaal JA, Van Hoof JP, Voorham J, Oostburg BF: Behavioral responses of Anopheles darlingi in Suriname to DDT residues on house walls. J Am Mosa Control Assoc 1989, 5:339-350.

122. Evans RG: Laboratory evaluation of the irritancy of bendiocarb, lambdacyhalothrin and DDT to Anopheles gambiae. J Am Mosa Control Assoc 1993, 9:285-293.

123. Chareonviriyaphap T, Aum-Aung B: An improved excito-repellency escape chamber for behavioral tests in mosquito vectors. Mekong Malaria Forum 2000, 5:82-87.

124. Chareonviriyaphap T, Prabaripai A, Sungvornyothrin S: An improved excitorepellency test chamber for mosquito behavioral tests. J Vector Ecol 2002, 27:250-252.

125. Chareonviriyaphap T, Sungvornyothin S, Ratanatham S, Prabaripai A: Insecticide-induced behavioral responses of Anopheles minimus, a malaria vector in Thailand. J Am Mosq Control Assoc 2001, 17:13-22.

126. Kongmee M, Prabaripai A, Akratanakul P, Bangs MJ, Chareonviriyaphap T: Behavioral responses of Aedes aegypti (Diptera: Culicidae) exposed to deltamethrin and possible implications for disease control. $J$ Med Entomol 2004, 41:1055-1063.

127. Potikasikorn J, Chareonviriyaphap T, Bangs MJ, Prabaripai A: Behavioral responses to DDT and pyrethroids between Anopheles minimus species A and C, malaria vectors in Thailand. Am J Trop Med Hyg 2005, 73:343-349.

128. Sungvornyothin S, Chareonviriyaphap T, Prabaripai A, Thirakhupt V, Ratanatham S, Bangs MJ: Effects of nutritional and physiological status on behavioral avoidance of Anopheles minimus (Diptera: Culicidae) to DDT, deltamethrin and lambdacyhalothrin. J Vector Ecol 2001, 26:202-215.

129. Grieco JP, Achee NL, Sardelis MR, Chauhan KR, Roberts DR: A novel high-throughput screening system to evaluate the behavioral response of adult mosquitoes to chemicals. J Am Mosq Control Assoc 2005, 21:404-411.

130. Roberts DR, Alecrim WD, Tavares AM, McNeill KM: Influence of physiological condition on the behavioral response of Anopheles darlingi to DDT. Mosq News 1984, 44:357-362.

131. Roberts DR, Alecrim WD, Tavares AM, Radke MG: The house-frequenting, host-seeking and resting behavior of Anopheles darlingi in southeastern Amazonas, Brazil. J Am Mosa Control Assoc 1987, 3:433-441.

132. Smith $A$ : A verandah-trap hut for studying the house-frequenting habits of mosquitos and for assessing insecticides. 2. The effect of dichlorvos (DDVP) on egress and mortality of Anopheles gambiae Giles and Mansonia uniformis (Theo.) entering naturally. Bull Entomol Res 1965, 56:275-282.
133. Bangs MJ: The susceptibility and behavior of Anopheles albimanus Weidemann and Anopheles vestitipennis Dyar \& Knab (Diptera: Culicidae) to insecticides in northern Belize, Central America. Bethesda, Maryland: Uniformed Services University of the Health Sciences; 1999:448.

134. Malaithong N, Polsomboon S, Poolprasert P, Parbaripai A, Bangs MJ, Suwonkerd W, Pothikasikorn J, Akratanakul P, Chareonviriyaphap T: Humanlanding patterns of Anopheles dirus sensu lato (Diptera: Culicidae) in experimental huts treated with DDT or deltamethrin. J Med Entomol 2010, 47:823-832.

135. Polsomboon S, Poolprasert P, Suwonkerd W, Bangs MJ, Tanasinchayakul S, Akratanakul P, Chareonviriyaphap T: Biting patterns of Anopheles minimus complex (Diptera: Culicidae) in experimental huts treated with DDT and deltamethrin. J Vector Ecol 2008, 33:285-292.

136. Roberts DR, Alecrim WD: Behavioral response of Anopheles darlingi to DDT-sprayed house walls in Amazonia. Bull Pan Am Health Organ 1991 25:210-217.

137. Muenworn V, Akaratanakul P, Bangs MJ, Parbaripai A, Chareonviriyaphap T: Insecticide-induced behavioral responses in two populations of Anopheles maculatus and Anopheles sawadwongporni, malaria vectors in Thailand. J Am Mosq Control Assoc 2006, 22:689-698.

138. Pothikasikorn J, Overgaard H, Ketavan C, Visetson S, Bangs MJ, Chareonviriyaphap T: Behavioral responses of malaria vectors, Anopheles minimus complex, to three classes of agrochemicals in Thailand. J Med Entomol 2007, 44:1032-1039.

139. Tisgratog R, Tananchai C, Bangs MJ, Tainchum K, Juntarajumnong W, Prabaripai A, Chauhan KR, Pothikasikorn J, Chareonviriyaphap T: Chemically induced behavioral responses in Anopheles minimus and Anopheles harrisoni in Thailand. J Vector Ecol 2011, 36:321-331.

140. Thanispong K, Achee NL, Bangs MJ, Grieco JP, Suwonkerd W, Prabaripai A, Chareonviriyaphap T: Irritancy and repellency behavioral responses of three strains of Aedes aegypti exposed to DDT and a-cypermethrin. J Med Entomol 2009, 46:1407-1414.

141. Mongkalangoon P, Grieco JP, Achee NL, Suwonkerd W, Chareonviriyaphap $\mathrm{T}$ : Irritability and repellency of synthetic pyrethroids on an Aedes aegypti population from Thailand. J Vector Ecol 2009, 34:217-224.

142. Thanispong K, Achee NL, Grieco JP, Bangs MJ, Suwonkerd W, Prabaripai A, Chauhan KR, Chareonviriyaphap T: A high throughput screening system for determining the three actions of insecticides against Aedes aegypti (Diptera: Culicidae) populations in thailand. J Med Entomol 2010, 47:833-841.

143. Muirhead-Thomson RC: The significance of irritability, behaviouristic avoidance and allied phenomena in malaria eradication. Bull World Health Organ 1960, 22:721-734.

144. Gahan JB, Lindquist AW: DDT residual sprays applied in buildings to control Anopheles quadrimaculatus. J Econ Entomol 1945, 38:223-230.

145. Metcalf RL, Hess AD, Smith GE, Jeffery GM, Ludwig GW: Observations on the use of DDT for the control of Anopheles quadrimaculatus. US Public Health Service; 1945:753-774. PMCID: PMC1976068

146. Trapido H: Recent experiments on possible resistance to DDT by Anopheles albimanus in Panama. Bull World Health Organ 1954 11:885-889.

147. Lindsay SW, Adiamah JH, Miller JE, Armstrong JR: Pyrethroid-treated bednet effects on mosquitoes of the Anopheles gambiae complex in The Gambia. Med Vet Entomol 1991, 5:477-483.

148. Miller JE: Laboratory and field studies of insecticide impregnated fibres for mosquito control, Ph.D. Thesis. London School of Hygiene and Tropical Medicine (University of London); 1990:336.

149. Killeen GF, Seyoum A, Sikaala C, Zomboko AS, Gimnig JE, Govella NJ, White MT: Eliminating malaria vectors. Parasit Vectors 2013, 6:172.

150. Georghiou GP: The evolution of resistance to pesticides. Annu Rev Ecol Syst 1972, 3:133-168.

151. Byford RL, Sparks TC: Chemical approaches to the management of resistant horn fly, Haematobia irritans (L.), populations. In Combating Resistance to Xenobiotics: Biological and Chemical Approaches. Edited by Ford MO, Holloman DW, Khambay BPS, Sawicki RM. Ellis Horwood: Chichester UK; 1987:178-189.

152. WHO: Resistance of vectors and reservoirs of disease to pesticides. In 737 TrotWECOVBaCTRSN. Geneva: World Health Organization; 1986.

153. Meyrowitsch DW, Pedersen EM, Alifrangis M, Scheike TH, Malecela MN, Magesa SM, Derua YA, Rwegoshora RT, Michael E, Simonsen PE: Is the 
current decline in malaria burden in sub-Saharan Africa due to a decrease in vector population? Malar J 2011, 10:188.

154. Killeen GF, Chitnis N, Moore SJ, Okumu FO: Target product profile choices for intra-domiciliary malaria vector control pesticide products: repel or kill? Malar J 2011, 10:207

155. Hiwat H, Hardjopawiro LS, Takken W, Villegas L: Novel strategies lead to pre-elimination of malaria in previously high-risk areas in Suriname. South America. Malar J 2012, 11:10. doi:10.1186/1475-2875-11-10.

156. Russell TL, Govella NJ, Azizi S, Drakeley CJ, Kachur SP, Killeen GF: Increased proportions of outdoor feeding among residual malaria vector populations following increased use of insecticide-treated nets in rural Tanzania. Malar J 2011, 6:80. doi:10.1186/1475-2875-10-80.

157. Mwangangi JM, Mbogo CM, Orindi BO, Muturi EJ, Midega JT, Nzovu J, Gatakaa H, Githure J, Borgemeister C, Keating J, Beier JC: Shifts in malaria vector species composition and transmission dynamics along the Kenyan coast over the past 20 years. Malar J 2013, 12:13. doi:10.1186/ 1475-2875-12-13

158. WHO: Guidelines for efficacy testing of spatial repellents. Control of Neglected Tropical Diseases, WHO Pesticide Evaluation Scheme. Geneva, Switzerland: World Health Organization; 2013. www.who.int/iris/bitstream/10665/78142/ 1/9789241505024_eng.pdf.

doi:10.1186/1756-3305-6-280

Cite this article as: Chareonviriyaphap et al:: Review of insecticide resistance and behavioral avoidance of vectors of human diseases in Thailand. Parasites \& Vectors 2013 6:280

\section{Submit your next manuscript to BioMed Central} and take full advantage of:

- Convenient online submission

- Thorough peer review

- No space constraints or color figure charges

- Immediate publication on acceptance

- Inclusion in PubMed, CAS, Scopus and Google Scholar

- Research which is freely available for redistribution 